\title{
A Joint Count-Continuous Model of Travel Behavior with Selection Based on a Multinomial Probit Residential Density Choice Model
}

Chandra R. Bhat (corresponding author)

The University of Texas at Austin

Department of Civil, Architectural and Environmental Engineering

301 E. Dean Keeton St. Stop C1761, Austin TX 78712

Phone: 512-471-4535; Fax: 512-475-8744

Email: bhat@mail.utexas.edu

and

King Abdulaziz University, Jeddah 21589, Saudi Arabia

\section{Sebastian Astroza}

The University of Texas at Austin

Department of Civil, Architectural and Environmental Engineering

301 E. Dean Keeton St. Stop C1761, Austin TX 78712

Phone: 512-471-4535, Fax: 512-475-8744

Email: sastroza@utexas.edu

\section{Raghuprasad Sidharthan}

The University of Texas at Austin

Department of Civil, Architectural and Environmental Engineering

301 E. Dean Keeton St. Stop C1761, Austin TX 78712

Phone: 512-471-4535, Fax: 512-475-8744

Email: raghu@mail.utexas.edu

Mohammad Jobair Bin Alam

King Abdulaziz University

Department of Civil Engineering

P.O. Box 80204, Jeddah 21589, Saudi Arabia

Phone: +966-2-6402000 (Ext.: 51339), Fax: +966-2-6952179

Email: malam@kau.edu.sa

Waleed H. Khushefati

King Abdulaziz University

Department of Civil Engineering

P.O. Box 80204, Jeddah 21589, Saudi Arabia

Phone: +966-2-6402000 (Ext.: 51339), Fax: +966-2-6952179

Email: wkhushefati@kau.edu.sa

July 2013 


\begin{abstract}
This paper formulates a multidimensional choice model system that is capable of handling multiple nominal variables, multiple count dependent variables, and multiple continuous dependent variables. The system takes the form of a treatment-outcome selection system with multiple treatments and multiple outcome variables. The Maximum Approximate Composite Marginal Likelihood (MACML) approach is proposed in estimation, and a simulation experiment is undertaken to evaluate the ability of the MACML method to recover the model parameters in such integrated systems. These experiments show that our estimation approach recovers the underlying parameters very well and is efficient from an econometric perspective. The parametric model system proposed in the paper is applied to an analysis of household-level decisions on residential location, motorized vehicle ownership, the number of daily motorized tours, the number of daily non-motorized tours, and the average distance for the motorized tours. The empirical analysis uses the NHTS 2009 data from the San Francisco Bay area. Model estimation results show that the choice dimensions considered in this paper are inter-related, both through direct observed structural relationships and through correlations across unobserved factors (error terms) affecting multiple choice dimensions. The significant presence of self-selection effects (endogeneity) suggests that modeling the various choice processes in an independent sequence of models is not reflective of the true relationships that exist across these choice dimensions, as also reinforced through the computation of treatment effects in the paper.
\end{abstract}

Keywords: multivariate dependency; self-selection; treatment effects; maximum approximate composite marginal likelihood; land-use and built environment; travel behavior 


\section{INTRODUCTION}

The focus on reducing traffic congestion, mobile-source emissions, greenhouse gas emissions, and fossil fuel dependence has led to the consideration of several travel demand management strategies at metropolitan planning organizations in the United States and other countries around the globe. While many of these strategies are based on economic incentives (and disincentives) to change autooriented travel behavior (originating from the concept of internalizing the full costs of auto travel), or on improved communication and technology-related policies to improve system performance in near real time, there also has been considerable interest in the land use-transportation planning connection, motivated by the possibility that land-use and urban form design policies can be used to control, manage, and shape individual traveler behavior and aggregate travel demand. Indeed, the literature on the subject is now vast and growing rapidly, with a substantial amount of quantitative and qualitative research having been undertaken in the past decade (see, for example, Bhat and Guo, 2007, Bhat et al., 2009, Transportation Research Board, 2009, Ewing and Cervero, 2012, Pinjari et al., 2011, Zhang et al., 2012, Handy and Krizek, 2012, and Bhat et al., 2013).

An issue that has received particular attention within the broad land use-transportation literature is whether any effect of the built environment on travel demand is causal or merely associative (or some combination of the two; see Bhat and Guo, 2007 and Mokhtarian and Cao, 2008). Commonly labeled as the residential self-selection problem, the underlying issue is that the data available to assess the potential effects of land-use on travel patterns is typically of a crosssectional nature. In such observational data, the residential location of households and the travel patterns of household members are jointly observed at a given point in time. Thus, the data reflects household residential location preferences co-mingled with the travel preferences of the households. On the other hand, from a policy perspective, the emphasis is on analyzing whether (and how much) a neo-urbanist design (compact built environment design, high bicycle lane and roadway street density, good land-use mix, and good transit and non-motorized mode accessibility/facilities) would help in reducing motorized vehicle miles of travel (VMT). To do so, the conceptual experiment that reveals the "true" effect of the built environment (BE) features of the residential location on travel patterns is the one that randomly locates households in residential locations. The problem then, econometrically speaking, is that the analyst has to extract out the "true" BE effect from a potentially non-randomly assigned (to residential locations) observed cross-sectional sample (in many metropolitan areas in the U.S., one could make a strong case for a non-random assignment of 
households to residential locations because of the relative undersupply of neo-urbanist development; see Levine, 2006). If the non-random assignment can be completely captured by observed non-travel characteristics of households and the BE (such as, say, poor households locating in areas with low housing cost), then a conventional travel model accommodating the observed non-travel characteristics of households and the BE characteristics would suffice to extract the "true" BE effect on travel (alternatively, one can use propensity-score techniques to match households based on observed non-travel characteristics and living in different neighborhoods, and compare the travel characteristics of these matched households to discern BE effects; see Cao and Fan, 2012). However, it is quite possible (if not likely) that there are some antecedent characteristics of households that are unobserved to the analyst and that impact both residential location choice and travel behavior. For instance, a household whose members have an overall auto inclination and a predisposition to enjoy private travel may locate itself in a conventional neighborhood (low population density, low bicycle lane and roadway street density, primarily single use residential land use, and auto-dependent urban design) and undertake substantial auto travel, while a household whose members dislike driving and prefer non-motorized and transit forms of travel may seek out neo-urbanist neighborhoods so they can pursue their activities using non-motorized and transit modes of travel. Ignoring such selfselection effects in residence choices can lead to a "spurious" causal effect of neighborhood attributes on travel, and potentially lead to misinformed BE design policies.

Many different approaches have been proposed in the literature to account for residential self-selection effects, a detailed review of which is beyond the scope of this paper (the reader is referred to Bhat and Guo, 2007, Bhat and Eluru, 2009, Mokhtarian and Cao, 2008, and Cao et al., 2009a). But, very broadly, one direction is to use longitudinal data to examine households that have moved residences, with the idea that the analyst can ostensibly control for the overall travel desires and attitudes of the members of a household, and attribute changes in travel behavior before and after a relocation to the different built environments in the two neighborhoods. However, even here, the analyst has to assume that the relocating household was in equilibrium in its pre-move neighborhood in terms of $\mathrm{BE}$ attributes, and moved because of factors unrelated to the preference for $\mathrm{BE}$ attributes (such as to upgrade the physical housing stock in response to higher incomes or a change in lifecycle). Another broad direction is to more explicitly capture what is traditionally "unobserved" in typical travel survey data sets, and include these as "observed" explanatory variables. Examples of these variables would be attitudes and perceptions related to travel, lifestyle, 
and $\mathrm{BE}$ attributes (such as importance of walking to daily activities, preference for open space and quietness, and level of interest in social interactions). Several studies in the literature have considered this approach, even as the field has seen an increasing movement in the past decade toward the use of a broader social-psychological framework and lense through which to observe and analyze travel-related behaviors (see, for example, van Acker et al., 2011, 2012, Spears et al., 2012, and Bamberg, 2013). This approach is a simple way of tackling the self-selection problem, but data on attitudes and perceptions are still not collected in large-scale MPO surveys. Besides, it is always possible that not all of the relevant attitudes/perceptions will be captured even in the most carefully designed survey to elicit such "soft" factors. A third broad direction is to accept the limitations of traditional cross-sectional surveys and attempt to control for self-selection effects through econometric instrumental variable techniques, and/or parametric distribution assumptions regarding the unobserved factors. Many earlier efforts in the transportation literature have used such an approach, which can also be used in combination with other approaches (see Chatman, 2009, Pinjari et al., 2011 and de Abreu e Silva et al., 2012). The current effort is along this third direction with important empirical extensions of earlier works as well as methodological innovations, as discussed in the next section.

\subsection{The Current Paper in the Context of Earlier Studies}

As discussed by Bhat and Guo (2007), there are several challenges in analyzing the effects of BE measures on travel behavior, even beyond the issue of residential self-selection, including the multidimensional nature of the $\mathrm{BE}$ and travel behavior. In terms of travel behavior, the different dimensions include motorized and non-motorized vehicle ownership by type, number of tours and stops, time-of-day, route choice, and travel mode choice. The net impact on overall VMT patterns will depend on the aggregation across the effects on individual travel dimensions. However, most earlier studies on the effect of BE measures on travel, while considering residential self-selection, focus directly (and solely) on the effect on vehicle miles of travel (see Zhang et al., 2012, Salon et al., 2012, and Cao and Fan, 2012, which are but a few recent examples). There have also been studies that consider residential self-selection and focus on BE effects on specific travel dimensions, such as auto ownership, vehicle type, trip frequencies, bicycle ownership, activity durations, and mode choice, though these have been relatively few and have focused on each dimension individually (see Bhat and Eluru, 2009 and Handy and Krizek, 2012 for detailed reviews). On the 
other hand, BE measures may have opposite effects on different dimensions characterizing the VMT components. For instance, a neo-urbanist design at the residence end may decrease trip lengths, but also increase the number of auto trips. As a result, a BE variable may appear to have no effect on VMT, though that may be because of opposite effects on different components constituting VMT. This is of relevance for policy, because the emissions per mile can be higher if a neo-urbanist design increases the number of auto trips, which may more than compensate for the emissions decrease because of a VMT decrease (see Sperry et al., 2012). Thus, there is a need to understand the differential effects of BE on different travel dimensions, rather than simply examine an aggregate effect on VMT or on an individual dimension of VMT. Further, the travel dimensions need to be modeled jointly because, as elucidated by Van Acker et al. (2012) and Paleti et al. (2013), selfselection need not be only through residential choice. For example, an auto-disinclined household may own fewer motorized vehicles, make fewer auto tours, as well as drive shorter distances using the car as the mode of transportation. As a consequence, any effect of the number of motorized vehicles on auto travel and VMT will be moderated by the auto-disinclined nature of the household. If some of the attributes associated with the auto-disinclined nature of the household are unobserved, there is self-selection in auto travel and VMT based not only on residential choice but also based on the number of motorized vehicles owned. This self-selection needs to be considered to obtain accurate estimates of BE effects and auto-ownership on travel-related attributes. That is, residential location may structurally affect motorized vehicle ownership and travel choices, and motorized vehicle ownership may structurally affect travel choices, but underlying propensities for vehicle ownership and travel choices may themselves affect residential location in the first place and underlying propensities for travel may affect motorized vehicle ownership. The only way to accurately reflect these impacts and capture the "bundling" of choices is to model the choice dimensions together in a joint equations modeling framework that accounts for correlated unobserved lifestyle (and other) effects as well as possible structural effects. ${ }^{1}$

\footnotetext{
${ }^{1}$ In joint limited-dependent variable systems in which one or more dependent variables are not observed on a continuous scale, such as the joint system considered in the current paper that has discrete dependent and count variables (which we will more generally refer to as limited-dependent variables), the structural effects of one limited-dependent variable on another can only be in a single direction. That is, it is not possible to have correlated unobserved effects underlying the propensities determining two limited-dependent variables, as well as have the observed limited-dependent variables themselves structurally affect each other in a bi-directional fashion. This creates a logical inconsistency problem (see Maddala, 1983, page 119 for a good discussion). Intuitively, the propensities are the precursors to the actual observed variables, and, when both the decisions are co-determined, it is impossible to have both observed variables structurally affect one another. In the current paper, we estimate models with each possible structural direction impact, and choose
} 
To be sure, there have been a few recent examples of a multi-dimensional modeling system in the land use-transportation literature. These systems use a two-stage instrumental variables approach (such as Vance and Hedel, 2007), or a structural equations approach (Van Acker et al., 2012 and de Abreu e Silva et al., 2012), or a simulated maximum likelihood or a simulated Bayesian inference approach (Eluru et al., 2010, Pinjari et al., 2011, and Brownstone and Golob, 2009). In the first (instrumental variable) approach, it can be a challenge to find good instruments; the approach also constitutes a limited information approach that can be fraught with econometric efficiency and collinearity problems (Puhani, 2000). The second (structural equations) approach and the third (simulation-based) approach, while plausible, do become cumbersome in the presence of a mixture of dependent variables (such as continuous, nominal, and count variables), and/or as the number of dimensions increases, as noted by earlier studies that use these approaches. In the current paper, we use the Maximum Approximate Composite Marginal Likelihood (MACML) approach proposed by Bhat (2011) that, in a relatively simple and practical manner, provides a way out to estimate multidimensional choice model systems. In this regard, the paper proposes the use of Bhat's MACML approach to estimate multi-dimensional systems with multiple nominal variables and multiple count dependent variables in the multi-dimensional system. In addition to providing a practical estimation approach, the approach is robust and yields consistent estimates under a range of possible full joint distributions that characterize the high-order dependency of endogenous variables in the multidimensional system. To our knowledge this is the first such sample selection formulation and application in the econometrics literature. In particular, the sample selection model takes the form of a treatment-outcome model with multiple treatments and multiple outcomes.

The parametric system proposed in this paper models residential choice as a discrete choice among a multinomial set of four land-use density categories as defined by housing unit density (housing units per square mile) within census blocks. This helps make the definition of choice alternatives clear and manageable, and also alleviates the problem of strong multi-collinearity of density with other $\mathrm{BE}$ characteristics that impact travel behavior. The use of density as the $\mathrm{BE}$ measure of interest is quite common, and has been used in many earlier residential self-selection

the one that provides a better data fit (which also turns out to be the one that is conceptually intuitive). However, it is critical to note that, regardless of which directionality of structural effects comes out to be better (or even if both directions are not statistically significant), the system is a joint bundled system because of the correlation in unobserved factors impacting the underlying propensities. 
studies, including the recent studies of Kim and Brownstone (2012), Paleti et al. (2013), and Cao and Fan (2012). The other endogenous variables in the system include the number of motorized vehicles in the household (a count variable), the number of motorized auto vehicle tours across all individuals in the household during the 24-hour period of the travel survey (another count variable), the number of non-motorized tours across all individuals in the household (a third count variable), and finally the continuous variable of average tour distance per auto tour. ${ }^{2}$ This last variable is obtained from the reported odometer readings before and after the day of the survey for each vehicle in the household. The natural logarithm of the average tour distance is used as the continuous dependent variable, after recoding the very small share of households with an average trip distance of less than 0.1 miles to 0.1 (so that the logarithmic dependent variable takes a real value for these households).

The key to our accommodation of count variables in the multi-dimensional system is the recasting of a univariate count model as a restricted version of a univariate generalized orderedresponse probit (GORP) model, as discussed in Castro, Paleti, and Bhat or CPB (2012). In addition to providing substantial flexibility to accommodate high or low probability masses for specific count outcomes, the latent variable-based count specification provides a convenient mechanism to tie the count outcomes with one another, and with the multinomial probit residential location choice model and the continuous average trip distance per auto trip model.

\section{MODEL STRUCTURE}

In this section, we first discuss the formulation for each type of variable, and then formulate the structure and estimation procedure for the multi-dimensional system.

\footnotetext{
${ }^{2}$ We focus on tours rather than trips to be consistent with an activity-based modeling framework that is increasingly being embraced by planning organizations. Besides, the focus on number of tours and tour distance brings in the decision component associated with episode chaining. Note also that it would be easy enough to extend the current framework to include the number of out-of-home episodes in the day as another count variable, or even the number of out-of-home episodes by purpose as multiple count outcomes, but we leave these for future exploration.
} 


\subsection{Nominal Dependent Variables}

Let there be $G$ nominal (unordered-response) variables for a household, and let $g$ be the index for the nominal variables $(g=1,2,3, \ldots, G)$. In the empirical context of the current paper, $G=1$ (the nominal variable is residential location). Also, let $I_{g}\left(I_{g} \geq 2\right)$ be the number of alternatives corresponding to the $g^{\text {th }}$ nominal variable and let $i_{g}$ be the corresponding index $\left(i_{g}=1,2,3, \ldots, I_{g}\right)$. Note that $I_{g}$ may vary across households, but the index for households is suppressed at this time for presentation convenience. We use a typical utility maximizing framework for the nominal variables, and write the utility for alternative $i_{g}$ for the $g$ th nominal variable as:

$$
U_{g i_{g}}=\boldsymbol{\beta}_{g}^{\prime} \boldsymbol{x}_{g i_{g}}+\varepsilon_{g i_{g}}
$$

where $\boldsymbol{x}_{g i_{g}}$ is a $\left(K_{g} \times 1\right)$-column vector of exogenous attributes as well as possibly the observed values of other endogenous nominal variables (introduced as dummy variables), other endogenous count variables, and other endogenous continuous variables. $\boldsymbol{\beta}_{g}$ is a $\left(K_{g} \times 1\right)$-column vector of corresponding coefficients, and $\varepsilon_{g i_{g}}$ is a normal error term. Let the variance-covariance matrix of the vertically stacked vector of errors $\varepsilon_{g}=\left[\left(\varepsilon_{g 1}, \varepsilon_{g 2}, \ldots, \varepsilon_{g I_{g}}\right)^{\prime}\right]$ be $\Lambda_{\mathrm{g}}$. The model above may be written in a more compact form by defining the following vectors and matrices: $\boldsymbol{U}_{g}=\left(U_{g 1}, U_{g 2}, \ldots, U_{g I_{g}}\right)^{\prime}$ ( $I_{g} \times 1$ vector), $\boldsymbol{x}_{\boldsymbol{g}}=\left(\boldsymbol{x}_{\boldsymbol{g} 1}, \boldsymbol{x}_{\boldsymbol{g} 2}, \boldsymbol{x}_{\boldsymbol{g} 3}, \ldots, \boldsymbol{x}_{\boldsymbol{g I}_{\boldsymbol{g}}}\right)^{\prime}\left(I_{g} \times K_{g}\right.$ matrix $)$, and $\boldsymbol{V}_{g}=\boldsymbol{x}_{\boldsymbol{g}} \boldsymbol{\beta}_{\boldsymbol{g}}\left(I_{g} \times 1\right.$ vector $)$. Then, $\boldsymbol{U}_{g} \sim M V N_{I_{g}}\left(\boldsymbol{V}_{g}, \boldsymbol{\Lambda}_{\mathrm{g}}\right)$, where $M V N_{I_{g}}\left(\boldsymbol{V}_{g}, \boldsymbol{\Lambda}_{\mathrm{g}}\right)$ is the multivariate normal distribution with mean vector $\boldsymbol{V}_{g}$ and covariance $\boldsymbol{\Lambda}_{\mathrm{g}}$. Consider now that the household chooses alternative $m_{g}$ for the $g$ th nominal variable. Under the utility maximization paradigm, $U_{g i_{g}}-U_{g m_{g}}$ must be less than zero for all $i_{g} \neq m_{g}$, since the household chose alternative $m_{g}$. Let $u_{g i_{g} m_{g}}^{*}=U_{g i_{g}}-U_{g m_{g}}\left(i_{g} \neq m_{g}\right)$, and stack the latent utility differentials into a vector $\boldsymbol{u}_{g}^{*}=\left[\left(u_{g 1 m_{g}}^{*}, u_{g 2 m_{g}}^{*}, \ldots, u_{g I_{g} m_{g}}^{*}\right)^{\prime} ; i_{g} \neq m_{g}\right]$.

In the context of the formulation above, several important identification issues need to be addressed (in addition to the usual identification consideration that one of the alternatives has to be used as the base for each nominal variable when introducing alternative-specific constants and variables that do not vary across the $I_{g}$ alternatives). First, only the covariance matrix of the error differences is estimable. Taking the difference with respect to the first alternative, only the elements 
of the covariance matrix $\breve{\Lambda}_{g}$ of $\varsigma_{g}=\left(\varsigma_{g 2}, \varsigma_{g 3}, \ldots, \varsigma_{g I_{g}}\right)$, where $\varsigma_{g i}=\varepsilon_{g i}-\varepsilon_{g 1}(i \neq 1)$, are estimable. However, the condition that $\boldsymbol{u}_{g}^{*}<\boldsymbol{0}_{I_{g}-1}$ takes the difference against the alternative $m_{g}$ that is chosen for the nominal variable $g$. Thus, during estimation, the covariance matrix $\vec{\Lambda}_{g}$ (of the error differences taken with respect to alternative $m_{g}$ is desired). Since $m_{g}$ will vary across households, $\vec{\Lambda}_{\mathrm{g}}$ will also vary across households. But all the $\vec{\Lambda}_{\mathrm{g}}$ matrices must originate in the same covariance matrix $\boldsymbol{\Lambda}_{g}$ for the original error term vector $\boldsymbol{\varepsilon}_{g}$. To achieve this consistency, $\boldsymbol{\Lambda}_{g}$ is constructed from $\breve{\Lambda}_{g}$ by adding an additional row on top and an additional column to the left. All elements of this

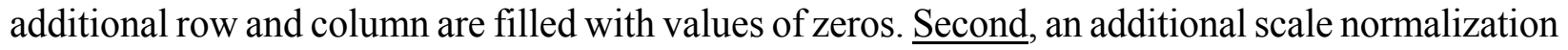
needs to be imposed on $\breve{\Lambda}_{g}$. For this, we normalize the first element of $\breve{\Lambda}_{g}$ to the value of one. Third, in MNP models, identification is tenuous when only household-specific covariates are used (see Keane, 1992 and Munkin and Trivedi, 2008). In particular, exclusion restrictions are needed in the form of at least one household characteristic being excluded from each alternative's utility in addition to being excluded from a base alternative (but appearing in some other utilities). Such exclusion restrictions may be identified based on the estimation of a simpler independent MNP model.

The discussion above focuses on a single nominal variable $g$. When there are $G$ nominal variables, define $\quad \vec{G}=\sum_{g=1}^{G} I_{g} \quad$ and $\quad \widetilde{G}=\sum_{g=1}^{G}\left(I_{g}-1\right) . \quad$ Further, let $\breve{\boldsymbol{u}}_{g}^{*}=\left(U_{g 2}-U_{g 1}, U_{g 3}-U_{g 1}, \ldots, U_{g I g}-U_{g 1}\right)^{\prime}, \quad \breve{\boldsymbol{u}}^{*}=\left(\left[\breve{\boldsymbol{u}}_{1}^{*}\right]^{\prime},\left[\breve{\boldsymbol{u}}_{2}^{*}\right]^{\prime}, \ldots,\left[\breve{\boldsymbol{u}}_{G}^{*}\right]^{\prime}\right)^{\prime}, \quad$ and $\boldsymbol{u}^{*}=\left(\left[\boldsymbol{u}_{1}^{*}\right]^{\prime},\left[\boldsymbol{u}_{2}^{*}\right]^{\prime}, \ldots,\left[\boldsymbol{u}_{G}^{*}\right]^{\prime}\right)^{\prime}$ (so $\breve{\boldsymbol{u}}^{*}$ is the vector of utility differences taken with respect to the first alternative for each nominal variable, while $\boldsymbol{u}^{*}$ is the vector of utility differences taken with respect to the chosen alternative for each nominal variable). Now, construct a matrix of dimension $\widetilde{G} \times \widetilde{G}$ that represents the covariance matrix of $\breve{\boldsymbol{u}}^{*}$ : 


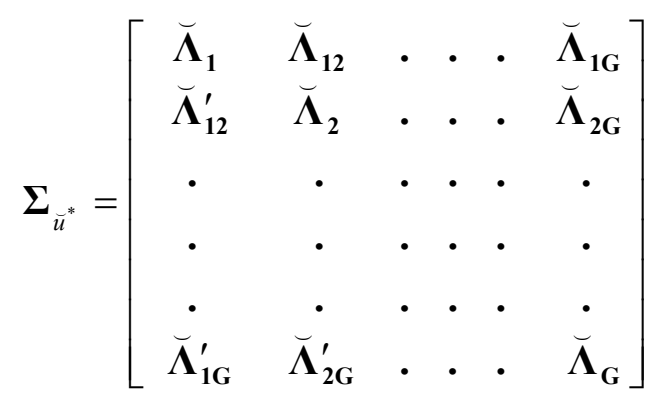

In the general case, this allows the estimation of $\sum_{g=1}^{G}\left(\frac{I_{g} *\left(I_{g}-1\right)}{2}-1\right)$ terms across all the $G$ nominal variables (originating from $\left(\frac{I_{g} *\left(I_{g}-1\right)}{2}-1\right)$ terms embedded in each $\breve{\Lambda}_{g}$ matrix; $\left.g=1,2, \ldots G\right)$ and the $\sum_{g=1}^{G-1} \sum_{l=g+1}^{G}\left(I_{g}-1\right) \times\left(I_{l}-1\right)$ covariance terms in the off-diagonal matrices of the $\boldsymbol{\Sigma}_{\tilde{u}^{*}}$ matrix characterizing the dependence between the latent utility differentials (with respect to the first alternative) across the nominal variables (originating from $\left(I_{g}-1\right) \times\left(I_{l}-1\right)$ estimable covariance terms within each off-diagonal matrix in $\boldsymbol{\Sigma}_{\vec{u}^{*}}$ ). For later use, define the stacked $\vec{G} \times 1-$ vectors $\boldsymbol{U}=\left(\boldsymbol{U}_{1}^{\prime}, \boldsymbol{U}_{2}^{\prime}, \ldots, \boldsymbol{U}_{G}^{\prime}\right)^{\prime}, \boldsymbol{V}=\left(\boldsymbol{V}_{1}^{\prime}, \boldsymbol{V}_{2}^{\prime}, \ldots, \boldsymbol{V}_{G}^{\prime}\right)^{\prime}$, and $\boldsymbol{\varepsilon}=\left(\varepsilon_{1}, \boldsymbol{\varepsilon}_{2}, \ldots, \varepsilon_{G}\right)^{\prime}$.

\subsection{Count Dependent Variables}

Let there be $L$ count variables for a household, and let $l$ be the index for the count variables $(l=1,2, \ldots, L)$. In the empirical context of the current paper, $L=3$ (the count variables are the number of motorized vehicles, the number of tours made by motorized vehicles, and the number of tours made by non-motorized forms of transportation). Let the count index be $j_{l}\left(j_{l}=0,1,2, \ldots, \infty\right)$ and let $n_{l}$ be the actual observed count value for the household. Then, a generalized version of the negative binomial model may be written in the form of a generalized ordered-response probit (GORP) formulation as:

$$
\begin{aligned}
& y_{l}^{*}=\xi_{l}, \quad j_{l}=n_{l} \text { if } \psi_{l, n_{l}-1}<y_{l}^{*}<\psi_{l, n_{l}}, \quad j_{l} \in\{0,1,2, \ldots . .\} \\
& \psi_{l, n_{l}}=\Phi^{-1}\left[\frac{\left(1-c_{l}\right)^{\theta_{l}}}{\Gamma\left(\theta_{l}\right)} \sum_{r=0}^{n_{l}}\left(\frac{\Gamma\left(\theta_{l}+r\right)}{r !} c_{l}^{r}\right)\right]+\varphi_{l, n_{l}}, c_{l}=\frac{\lambda_{l}}{\lambda_{l}+\theta_{l}} \text {, and } \lambda_{l}=e^{\mu_{l}^{\prime} z_{l}} .
\end{aligned}
$$


In the above equation, $y_{l}^{*}$ is a latent continuous stochastic propensity variable associated with count variable $l$ that maps into the observed count $n_{l}$ through the $\psi_{l}$ vector (which is a vertically stacked column vector of thresholds $\left(\psi_{l,-1}, \psi_{l, 0}, \psi_{l, 1}, \psi_{l, 2}, \ldots\right)^{\prime}$. This variable, which is equated to $\xi_{l}$ in the GORP formulation above, is a standard normal random error term. $\boldsymbol{\mu}_{\boldsymbol{l}}$ is a column vector corresponding to another vector $\boldsymbol{z}_{l}$ (including a constant) of exogenous observable covariates as well as possibly the observed values of other endogenous variables. $\Phi^{-1}$ in the threshold function of Equation (3) is the inverse function of the univariate cumulative standard normal. $\theta_{l}$ is a parameter that provides flexibility to the count formulation, and is related to the dispersion parameter in a traditional negative binomial model $\left(\theta_{l}>0 \forall l\right) . \Gamma\left(\theta_{l}\right)$ is the traditional gamma function; $\Gamma\left(\theta_{l}\right)=\int_{0}^{\infty} t^{\theta_{l}-1} e^{-t} d t$. The threshold terms in the $\psi_{l}$ vector satisfy the ordering condition (i.e., $\left.\psi_{l,-1}<\psi_{l, 0}<\psi_{l, 1}<\psi_{l, 2} \ldots .<\infty \forall l\right)$ as long as $\varphi_{l,-1}<\varphi_{l, 0}<\varphi_{l, 1}<\varphi_{l, 2} \ldots .<\infty{ }^{3}$ The presence of the $\varphi_{l}$ terms in the thresholds provides substantial flexibility to accommodate high or low probability masses for specific count outcomes without the need for cumbersome traditional treatments using zero-inflated or related mechanisms in multi-dimensional model systems. For identification, we set $\varphi_{l,-1}=-\infty, \psi_{l,-1}=-\infty$, and $\varphi_{l, 0}=0$ for all count variables $l$. In addition, we identify a count value $e_{l}^{*} \quad\left(e_{l}^{*} \in\{0,1,2, \ldots \ldots\}\right)$ above which $\varphi_{l, e}(e \in\{0,1,2, \ldots \ldots\})$ is held fixed at $\varphi_{l, e_{l}^{*}}$; that is, $\varphi_{l, e}=\varphi_{l, e_{l}^{*}}$ if $e_{l}>e_{l}^{*}$, where the value of $e_{l}^{*}$ can be based on empirical testing. For later use, let $\boldsymbol{\varphi}_{l}=\left(\varphi_{l, 1}, \varphi_{l, 2}, \ldots \varphi_{l, e^{*}}\right)^{\prime}\left(e_{l}^{*} \times 1\right.$ vector $)$, and $\boldsymbol{\varphi}=\left(\boldsymbol{\varphi}_{1}^{\prime}, \boldsymbol{\varphi}_{2}^{\prime}, \ldots \boldsymbol{\varphi}_{L}^{\prime}\right)^{\prime}$. Also, stack the $L$ latent variables $y_{l}^{*}$ into an $(L \times 1)$ vector $\boldsymbol{y}^{*}$, and let $\boldsymbol{y}^{*} \sim M V N_{L}\left(\boldsymbol{f}, \boldsymbol{\Sigma}_{y^{*}}\right)$, where $\boldsymbol{f}=\boldsymbol{0}_{L}$ and $\boldsymbol{\Sigma}_{y^{*}}$ is the covariance (correlation) matrix of $\boldsymbol{\xi}=\left(\xi_{1}, \xi_{2}, \ldots, \xi_{L}\right)$. Also, stack the lower thresholds $\psi_{l, n_{l}-1}(l=1,2, \ldots, L)$ into an $(L \times 1)$ vector $\psi_{l o w}$ and the upper thresholds $\psi_{l, n_{l}}(l=1,2, \ldots, L)$ into another vector $\psi_{u p} \cdot 4$

\footnotetext{
${ }^{3}$ The nature of the functional form for the non- $\varphi$ component of the thresholds satisfy the ordering conditions by construction.

${ }^{4}$ The specification of the GORP model in Equation (3) provides a flexible mechanism to model count data. It subsumes the traditional count models as specific and restrictive cases. In particular, if all elements of the $\varphi_{I}$ vector are zero, the model in Equation (3) for count variable $l$ collapses to a univariate traditional negative binomial model with dispersion parameter $\theta_{l}$. If, in addition, $\theta_{l} \rightarrow \infty$, the result is the Poisson count model.
} 


\subsection{Continuous Dependent Variables}

Finally, let there be $H$ continuous variables $\left(y_{1}, y_{2}, \ldots, y_{H}\right)$ with an associated index $h$ $(h=1,2, \ldots, H)$. In the empirical context of the current paper, $H=1$ (the continuous variable is the natural logarithm of average tour distance). Let $y_{h}=\gamma_{h}^{\prime} \boldsymbol{s}_{h}+\eta_{h}$ in the usual linear regression fashion, where the vector $\boldsymbol{s}_{h}$ includes exogenous household variables as well as possibly other endogenous variables. Stacking the $H$ continuous variables into a $(H \times 1)$-vector $\boldsymbol{y}$, one may write $\boldsymbol{y}=\operatorname{MVN}_{h}\left(\boldsymbol{d}, \boldsymbol{\Sigma}_{y}\right)$, where $\boldsymbol{d}=\left(\boldsymbol{\gamma}_{1}^{\prime} \boldsymbol{s}_{1}, \boldsymbol{\gamma}_{2}^{\prime} \boldsymbol{s}_{2}, \ldots . . \gamma_{H}^{\prime} \boldsymbol{s}_{\boldsymbol{H}}\right)^{\prime}$, and $\boldsymbol{\Sigma}_{y}$ is the covariance matrix of $\boldsymbol{\eta}=\left(\eta_{1}, \eta_{2}, \ldots . \eta_{H}\right)$.

\subsection{The Joint Model System and Likelihood Formation}

The jointness across the different types of dependent variables may be specified by writing the covariance matrix of $\breve{\boldsymbol{y}}=\left(\breve{\boldsymbol{u}}^{*}, \boldsymbol{y}^{*}, \boldsymbol{y}\right)$ as:

$\operatorname{Var}(\breve{\boldsymbol{y}})=\breve{\boldsymbol{\Omega}}=\left[\begin{array}{ccc}\boldsymbol{\Sigma}_{\breve{u}^{*}} & \boldsymbol{\Sigma}_{\breve{u}^{*} y^{*}} & \boldsymbol{\Sigma}_{\vec{u}^{*} y} \\ \boldsymbol{\Sigma}_{\breve{u}^{*} y^{*}}^{\prime} & \boldsymbol{\Sigma}_{y^{*}} & \boldsymbol{\Sigma}_{y^{*} y} \\ \boldsymbol{\Sigma}_{\breve{u}^{*} y}^{\prime} & \boldsymbol{\Sigma}_{y^{*} y}^{\prime} & \boldsymbol{\Sigma}_{y}\end{array}\right]$,

where $\boldsymbol{\Sigma}_{\breve{u}^{*} y^{*}}$ is a $\widetilde{G} \times L$ matrix capturing covariance effects between the $\breve{\boldsymbol{u}}^{*}$ vector and the $\boldsymbol{y}^{*}$ vector, $\boldsymbol{\Sigma}_{\breve{u}^{*} \mathrm{y}}$ is a $\widetilde{G} \times H$ matrix capturing covariance effects between the $\breve{\boldsymbol{u}}^{*}$ vector and the $\boldsymbol{y}$ vector, and $\boldsymbol{\Sigma}_{y^{*} y}$ is an $L \times H$ matrix capturing covariance effects between the $\boldsymbol{y}^{*}$ vector and the $\boldsymbol{y}$ vector. All elements of the matrix above are identifiable. However, the matrix represents the covariance of latent utility differentials taken with respect to the first alternative for each of the nominal variables. For estimation, the corresponding matrix with respect to the latent utility differentials with respect to the chosen alternative for each nominal variable, say $\widetilde{\mathbf{\Omega}}$, is needed. For this purpose, first construct the general covariance matrix $\boldsymbol{\Omega}$ for the original $[\vec{G}+L+H] \times 1$ vector $\boldsymbol{U} \boldsymbol{Y}=\left(\boldsymbol{U}^{\prime}, \boldsymbol{y}^{*^{\prime}}, \boldsymbol{y}^{\prime}\right)^{\prime}$, while also ensuring all parameters are identifiable (note that $\mathbf{\Omega}$ is equivalently the covariance matrix of $\boldsymbol{\tau}=\left(\varepsilon^{\prime}, \xi^{\prime}, \eta^{\prime}\right)^{\prime}$, which we will use in the simulation section). To do so, define a matrix $\boldsymbol{D}$ of size 
$[\vec{G}+L+H] \times[\widetilde{G}+L+H]$. The first $I_{1}$ rows and $\left(I_{1}-1\right)$ columns correspond to the first nominal variable. Insert an identity matrix of size $\left(I_{1}-1\right)$ after supplementing with a first row of zeros in the first through $\left(I_{1}-1\right)$ th columns of the matrix. The rest of the elements in the first $I_{1}$ rows and the first $\left(I_{1}-1\right)$ columns take a value of zero. Next, rows $\left(I_{1}+1\right)$ through $\left(I_{1}+I_{2}\right)$ and columns $\left(I_{1}\right)$ through $\left(I_{1}+I_{2}-2\right)$ correspond to the second nominal variable. Again position an identity matrix of size $\left(I_{2}-1\right)$ after supplementing with a first row of zeros into this position. Continue this for all $G$ nominal variables. Put zero values in all cells without any value up to this point. Finally, insert an identity matrix of size $L+H$ into the last $L+H$ rows and $L+H$ columns of the matrix $\boldsymbol{D}$. Thus, for the case with two nominal variables, one nominal variable with 3 alternatives and the second with four alternatives, one count variable, and one continuous variable, the matrix $\boldsymbol{D}$ takes the form shown below:

$$
\left[\begin{array}{cc:ccr:cr}
0 & 0 & 0 & 0 & 0 & 0 & 0 \\
1 & 0 & 0 & 0 & 0 & 0 & 0 \\
0 & 1 & 0 & 0 & 0 & 0 & 0 \\
\hdashline 0 & 0 & 0 & 0 & 0 & 0 & 0 \\
0 & 0 & 1 & 0 & 0 & 0 & 0 \\
0 & 0 & 0 & 1 & 0 & 0 & 0 \\
0 & 0 & 0 & 0 & 1 & 0 & 0 \\
\hdashline 0 & 0 & 0 & 0 & 0 & 1 & 0 \\
0 & 0 & 0 & 0 & 0 & 0 & 1
\end{array}\right]_{9 * 7}
$$

Then, the general covariance matrix of $\boldsymbol{U} \boldsymbol{Y}$ may be developed as $\boldsymbol{\Omega}=\mathbf{D} \boldsymbol{\Omega} \mathbf{D}^{\prime}$. All parameters in this matrix are identifiable by virtue of the way this matrix is constructed based on utility differences and, at the same time, it provides a consistent means to obtain the covariance matrix $\widetilde{\mathbf{\Omega}}$ that is needed for estimation (and is with respect to each individual's chosen alternative for each nominal variable). Specifically, to develop the distribution for the vector $\tilde{\boldsymbol{y}}=\left(\boldsymbol{u}^{*}, \boldsymbol{y}^{*^{\prime}}, \boldsymbol{y}^{\prime}\right)^{\prime}$, define a matrix $\boldsymbol{M}$ of size $[\widetilde{G}+L+H] \times[\overleftrightarrow{G}+L+H]$. The first $\left(I_{1}-1\right)$ rows and $I_{1}$ columns correspond to the first nominal variable. Insert an identity matrix of size $\left(I_{1}-1\right)$ after supplementing with a column of ' -1 ' values in the column corresponding to the chosen alternative. The rest of the columns for the first 
$\left(I_{1}-1\right)$ rows and the rest of the rows for the first $I_{1}$ columns take a value of zero. Next, rows $\left(I_{1}\right)$ through $\left(I_{1}+I_{2}-2\right)$ and columns $\left(I_{1}+1\right)$ through $\left(I_{1}+I_{2}\right)$ correspond to the second nominal variable. Again position an identity matrix of size $\left(I_{2}-1\right)$ after supplementing with a column of '-1' values in the column corresponding to the chosen alternative. Continue this procedure for all $G$ nominal variables. Finally, insert an identity matrix of size $L+H$ into the last $L+H$ rows and $L+H$ columns of the matrix $\boldsymbol{M}$. With the matrix $\boldsymbol{M}$ as defined, the covariance matrix $\widetilde{\mathbf{\Omega}}$ is given by $\widetilde{\mathbf{\Omega}}=\mathbf{M} \mathbf{\Omega} \mathbf{M}^{\prime}$.

Next, define $\widetilde{\boldsymbol{u}}=\left(\boldsymbol{u}^{* \prime}, \boldsymbol{y}^{* \prime \prime}\right)^{\prime}$ and $\widetilde{\boldsymbol{g}}=\left((\boldsymbol{M} \boldsymbol{V})^{\prime}, \boldsymbol{f}^{\prime}\right)^{\prime}$. Also, partition $\widetilde{\boldsymbol{\Omega}}$ so that $\widetilde{\boldsymbol{\Omega}}=\left[\begin{array}{ccc}\widetilde{\boldsymbol{\Sigma}}_{u^{*}} & \widetilde{\boldsymbol{\Sigma}}_{u^{*} y^{*}} & \widetilde{\boldsymbol{\Sigma}}_{u^{*} y} \\ \widetilde{\boldsymbol{\Sigma}}_{u^{*} y^{*}}^{\prime} & \boldsymbol{\Sigma}_{y^{*}} & \boldsymbol{\Sigma}_{y^{*} y} \\ \widetilde{\boldsymbol{\Sigma}}_{u^{*} y}^{\prime} & \boldsymbol{\Sigma}_{y^{*} y}^{\prime} & \boldsymbol{\Sigma}_{y}\end{array}\right]$

Let $\widetilde{\boldsymbol{\Sigma}}_{\widetilde{u}}=\left[\begin{array}{cc}\widetilde{\boldsymbol{\Sigma}}_{u^{*}} & \widetilde{\boldsymbol{\Sigma}}_{u^{*} y^{*}} \\ \widetilde{\boldsymbol{\Sigma}}_{u^{*} \mathbf{y}^{*}}^{\prime} & \boldsymbol{\Sigma}_{\mathbf{y}^{*}}\end{array}\right]$ and $\operatorname{Var}(\widetilde{\boldsymbol{y}})=\widetilde{\boldsymbol{\Omega}}=\left[\begin{array}{cc}\widetilde{\boldsymbol{\Sigma}}_{\widetilde{u}} & \widetilde{\boldsymbol{\Sigma}}_{\widetilde{u} y} \\ \widetilde{\boldsymbol{\Sigma}}_{\tilde{u} y}^{\prime} & \boldsymbol{\Sigma}_{y}\end{array}\right]$, where $\widetilde{\boldsymbol{\Sigma}}_{\widetilde{u} y}=\left[\begin{array}{c}\widetilde{\boldsymbol{\Sigma}}_{u^{*} y} \\ \boldsymbol{\Sigma}_{y^{*} y}\end{array}\right](\widetilde{G}+L) \times H$ matrix. Also, supplement the threshold vectors defined earlier as follows: $\widetilde{\psi}_{\text {low }}=\left[\left(-\infty_{\widetilde{G}}\right)^{\prime}, \boldsymbol{\psi}_{\text {low }}^{\prime}\right]$, and $\widetilde{\boldsymbol{\psi}}_{u p}=\left[\left(\boldsymbol{\boldsymbol { o }}_{\widetilde{G}}\right)^{\prime}, \boldsymbol{\psi}_{u p}^{\prime}\right]$, where $-\infty_{\widetilde{G}}$ is a $(\widetilde{G} \times 1)$-column vector of negative infinities, and $\boldsymbol{\boldsymbol { 0 }}_{\widetilde{G}}$ is another $(\widetilde{G} \times 1)$-column vector of zeros. The conditional distribution of $\widetilde{\boldsymbol{u}}$ given $\boldsymbol{y}$, is multivariate normal with mean $\widetilde{\boldsymbol{g}}=\widetilde{\boldsymbol{g}}+\widetilde{\boldsymbol{\Sigma}}_{\tilde{u} y} \boldsymbol{\Sigma}_{y}^{-1}(\boldsymbol{y}-\boldsymbol{d})$ and variance $\widetilde{\widetilde{\boldsymbol{\Sigma}}}_{\tilde{u}}=\widetilde{\boldsymbol{\Sigma}}_{\tilde{u}}-\widetilde{\boldsymbol{\Sigma}}_{\tilde{u} y} \boldsymbol{\Sigma}_{y}^{-1} \widetilde{\boldsymbol{\Sigma}}_{\tilde{u} y}^{\prime}$.

Next, let $\boldsymbol{\theta}$ be the collection of parameters to be estimated: $\boldsymbol{\theta}=\left[\boldsymbol{\beta}_{1}, \ldots, \boldsymbol{\beta}_{G} ; \boldsymbol{\mu}_{1}, \ldots \boldsymbol{\mu}_{L} ; \boldsymbol{\varphi}_{1}, \ldots, \boldsymbol{\varphi}_{L ;} ; \boldsymbol{\gamma}_{1}, \ldots, \boldsymbol{\gamma}_{H} ; \operatorname{Vech}(\breve{\boldsymbol{\Omega}})\right]$, where $\operatorname{Vech}(\breve{\boldsymbol{\Omega}})$ represents the vector of upper triangle elements of $\breve{\boldsymbol{\Omega}}$. Then the likelihood function for the household may be written as:

$$
\begin{aligned}
L(\boldsymbol{\theta}) & =\phi_{H}\left(\boldsymbol{y}-\boldsymbol{d} \mid \boldsymbol{\Sigma}_{y}\right) \times \operatorname{Pr}\left[\widetilde{\boldsymbol{\psi}}_{l o w} \leq \widetilde{\boldsymbol{u}} \leq \widetilde{\boldsymbol{\psi}}_{u p}\right], \\
& =\phi_{H}\left(\boldsymbol{y}-\boldsymbol{d} \mid \boldsymbol{\Sigma}_{y}\right) \times \int_{D_{\tilde{u}}} \phi_{\widetilde{G}+L}\left(\widetilde{\boldsymbol{u}} \mid \widetilde{\boldsymbol{g}}, \widetilde{\boldsymbol{\Sigma}}_{\widetilde{u}}\right) d \widetilde{u},
\end{aligned}
$$

where the integration domain $D_{\tilde{u}}=\left\{\widetilde{\boldsymbol{u}}: \widetilde{\boldsymbol{\psi}}_{\text {low }} \leq \widetilde{\boldsymbol{u}} \leq \widetilde{\boldsymbol{\psi}}_{\text {up }}\right\}$ is simply the multivariate region of the elements of the $\widetilde{\boldsymbol{u}}$ vector determined by the range $(-\infty, 0)$ for the nominal variables and by the 
observed outcomes of the ordinal variables, and $\phi_{\widetilde{G}+L}($.$) is the multivariate normal density function$ of dimension $\widetilde{G}+L$. The likelihood function for a sample of $Q$ households is obtained as the product of the household-level likelihood functions.

The above likelihood function involves the evaluation of a $\widetilde{G}+L$-dimensional rectangular integral for each household, which can be computationally expensive if there are several nominal variables, or if each nominal variable takes a large number of values, or if there are several count variables, or combinations of these. So, the Maximum Approximate Composite Marginal Likelihood (MACML) approach of Bhat (2011), in which the likelihood function only involves the computation of univariate and bivariate cumulative distributive functions, is used in this paper.

\subsection{The MACML Estimation Approach}

The MACML approach combines a composite marginal likelihood (CML) estimation approach with an approximation method to evaluate the multivariate standard normal cumulative distribution (MVNCD) function. The MACML approach, similar to the parent CML approach (see Varin et al., 2011 for a recent review of CML approaches), maximizes a surrogate likelihood function that compounds much easier-to-compute, lower-dimensional, marginal likelihoods (see Varin et al., 2011 for a recent extensive review of CML methods; Lindsay et al., 2011, Bhat, 2011, and Yi et al., 2011 are also useful references). The CML approach, which belongs to the more general class of composite likelihood function approaches (see Lindsay, 1988), may be explained in a simple manner as follows. In the multi-dimensional model, instead of developing the likelihood function for the entire set of dimensions at once, as in Equation (6), one may compound (multiply) pairwise probabilities of each pair of non-continuous dimensions for the household. The CML estimator (in this instance, the pairwise CML estimator) is then the one that maximizes the compounded probability of all pairwise events. The properties of the CML estimator may be derived using the theory of estimating equations (see Cox and Reid, 2004, Yi et al., 2011). Specifically, under usual regularity assumptions (Molenberghs and Verbeke, 2005, page 191, Xu and Reid, 2011), the CML estimator is consistent and asymptotically normal distributed (this is because of the unbiasedness of the CML score function, which is a linear combination of proper score functions associated with the marginal event probabilities forming the composite likelihood; for a formal proof, see Yi et al., 2011 and $\mathrm{Xu}$ and Reid, 2011). Further, the CML approach is robust against mis-specification of the full 
joint distribution of the endogenous variables in the multi-dimensional system, while the traditional maximum likelihood approach is not (Xu and Reid, 2011). In particular, the consistency of the estimates in the CML approach is predicated only on the correct specification of the lower dimensional marginal densities appearing in the CML function, without any need for explicit distributional assumptions for the full dimensional density of the multi-dimensional system. This is a particularly attractive feature of the CML inference approach when modeling high dimensional econometric systems, because mis-specifications of the full dimensional joint density function are much more likely than mis-specifications of lower dimensional densities.

In the MACML approach, the MVNCD function appearing in the CML function is evaluated using an analytic approximation method rather than simulation techniques. This combination of the CML with the specific analytic approximation for the MVNCD function is effective because it involves only univariate and bivariate cumulative normal distribution function evaluations. The MVNCD approximation method is based on linearization with binary variables (see Bhat, 2011). As has been demonstrated by Bhat and Sidharthan (2012), the MACML method has the virtue of computational robustness in that the approximate CML surface is smoother and easier to maximize than traditional simulated maximum likelihood surfaces.

In the context of the proposed model, consider the following (pairwise) composite marginal likelihood function formed by taking the products (across the $G$ nominal variables and $L$ count variables) of the joint pairwise probability of the chosen alternatives for a household.

$$
\begin{aligned}
L_{C M L}(\boldsymbol{\theta})=\phi_{H}\left(\boldsymbol{y}-\boldsymbol{d} \mid \boldsymbol{\Sigma}_{\mathbf{y}}\right) \times( & \left.\prod_{g=1}^{G-1} \prod_{g^{\prime}=g+1}^{G} \operatorname{Pr}\left(d_{i_{g}}=m_{g}, d_{i_{g^{\prime}}}=m_{g^{\prime}}\right)\right) \times\left(\prod_{l=1}^{L-1} \prod_{l^{\prime}=l+1}^{L} \operatorname{Pr}\left(j_{l}=n_{l}, j_{l^{\prime}}=n_{i^{\prime}}\right)\right) \\
& \times\left(\prod_{g=1}^{G} \prod_{l^{\prime}=1}^{L} \operatorname{Pr}\left(d_{i_{g}}=m_{g}, j_{l}=n_{l}\right)\right) .
\end{aligned}
$$

where $d_{i_{g}}$ is an index for the individual's choice for the $g^{\text {th }}$ nominal variable. The net result is that the pairwise likelihood function now only needs the evaluation of $\widetilde{G}_{g g^{\prime}}, \widetilde{G}_{l l^{\prime}}$, and $\widetilde{G}_{g l}$ dimensional cumulative normal distribution functions (rather than the $\widetilde{G}+L$-dimensional cumulative distribution function in the maximum likelihood function), where $\widetilde{G}_{g g^{\prime}}=I_{g}+I_{g^{\prime}}-2, \widetilde{G}_{l l^{\prime}}=2$, and $\widetilde{G}_{g l}=I_{g}$. This leads to substantial computational efficiency. However, in cases where there are several alternatives for one or more nominal variables, the dimension $\widetilde{G}_{g g^{\prime}}$ and $\widetilde{G}_{g l}$ can still be quite high. This is where the use of an analytic approximation of the multivariate normal cumulative distribution (MVNCD) 
function, as shown in Bhat (2011), is convenient. Also note that the probabilities in the CML function in Equation (7) can be computed by selecting out the appropriate sub-matrices of the mean

vector $\widetilde{\boldsymbol{g}}$ and the covariance matrix $\widetilde{\widetilde{\boldsymbol{\Sigma}}}_{\widetilde{u}}$ of the vector $\widetilde{\boldsymbol{u}}$, and the appropriate sub-vectors of the threshold vectors $\widetilde{\boldsymbol{\psi}}_{\text {low }}$ and $\widetilde{\boldsymbol{\psi}}_{\text {up }}$. The covariance matrix of the parameters $\boldsymbol{\theta}$ may be estimated by the inverse of Godambe's (1960) sandwich information matrix (see Zhao and Joe, 2005).

$V_{M A C M L}(\boldsymbol{\theta})=[G(\boldsymbol{\theta})]^{-1}=H(\boldsymbol{\theta})[J(\boldsymbol{\theta})]^{-1}[H(\boldsymbol{\theta})]$,

$H(\boldsymbol{\theta})$ and $J(\boldsymbol{\theta})$ can be estimated in a straightforward manner at the MACML estimate $\hat{\boldsymbol{\theta}}_{\boldsymbol{M A C M \boldsymbol { L }}}$ as follows (introducing $q$ as the index for households):

$$
\begin{aligned}
& \hat{H}(\hat{\boldsymbol{\theta}})=-\left[\sum_{q=1}^{Q} \frac{\partial^{2} \log L_{M A C M L, q}(\boldsymbol{\theta})}{\partial \boldsymbol{\theta} \partial \boldsymbol{\theta}^{\prime}}\right]_{\hat{\boldsymbol{\theta}}_{M A C M L}}, \text { and } \\
& \hat{J}(\hat{\boldsymbol{\theta}})=\sum_{q=1}^{Q}\left[\left(\frac{\partial \log L_{M A C M L, q}(\boldsymbol{\theta})}{\partial \boldsymbol{\theta}}\right)\left(\frac{\partial \log L_{M A C M L, q}(\boldsymbol{\theta})}{\partial \boldsymbol{\theta}^{\prime}}\right)\right]_{\hat{\theta}_{M A C M L}} .
\end{aligned}
$$

\subsection{Positive Definiteness}

The matrix $\widetilde{\boldsymbol{\Omega}}$ for each household has to be positive definite. The simplest way to guarantee this is to ensure that the matrix $\breve{\mathbf{\Omega}}$ is positive definite. To do so, the Cholesky matrix of $\breve{\mathbf{\Omega}}$ may be used as the matrix of parameters to be estimated. However, note that the top diagonal element of each $\breve{\Lambda}_{g}$ in $\breve{\mathbf{\Omega}}$ is normalized to one for identification, and this restriction should be recognized when using the Cholesky factor of $\breve{\boldsymbol{\Omega}}$. Further, the diagonal elements of $\boldsymbol{\Sigma}_{y^{*}}$ in $\breve{\boldsymbol{\Omega}}$ are also normalized to one. These restrictions can be maintained by appropriately parameterizing the diagonal elements of the Cholesky decomposition matrix. Thus, consider the lower triangular Cholesky matrix $\breve{\boldsymbol{L}}$ of the same size as $\breve{\mathbf{\Omega}}$. Whenever a diagonal element (say the $k k^{\text {th }}$ element) of $\breve{\mathbf{\Omega}}$ is to be normalized to one, the corresponding diagonal element of $\breve{\boldsymbol{L}}$ is written as $\sqrt{1-\sum_{j=1}^{a-1} d_{k j}^{2}}$, where the $d_{k j}$ elements are the

Cholesky factors that are to be estimated. With this parameterization, $\breve{\boldsymbol{\Omega}}$ obtained as $\breve{\boldsymbol{L}} \breve{\boldsymbol{L}}^{\prime}$ is positive definite and adheres to the scaling conditions. 


\section{SIMULATION STUDY}

The simulation exercise undertaken in this section examines the ability of the MACML estimator to recover parameters from finite samples in the joint model by generating simulated data sets with known underlying model parameters. We consider a single nominal variable with three alternatives, a single count variable, and a single continuous variable.

\subsection{Experimental Design}

Assume a single independent variable for each of the three alternatives in the MNP model for the nominal choice. The values of this variable for each alternative are drawn from a standard univariate normal distribution to construct a synthetic sample of 2000 realizations of the exogenous variable $(Q=2000)$. The coefficient on this variable (labeled as $\beta$ ) is set to the value of -1 . For the count variable, we consider an exogenous variable in the $z_{l}$ vector (embedded in the threshold function), generated again from a standard univariate distribution. The corresponding coefficient (labeled as $\mu_{1}$ ) is set to 0.5 . In addition, dummy variables corresponding to the choice of the second alternative and third alternative in the nominal variable are included as structural effects in the count specification through the $z_{l}$ vector, with coefficients of $\mu_{2}=0.25$ and $\mu_{3}=0.5$. The dispersion parameter $\theta_{l}$ (or simply $\theta$ in this section) is fixed at 2 , and the $\varphi_{l}=\left(\varphi_{l, 1}, \varphi_{l, 2}, \ldots \varphi_{l, e^{*}}\right)^{\prime}$ vector (labeled $\boldsymbol{\varphi}$ here) is set so that $\boldsymbol{\varphi}=\left(\varphi_{1}, \varphi_{2}\right)=(0.3,0.6)$. For the continuous variable, a single standard normally distributed variable is generated with a coefficient of $\gamma=2$, with no additional structural effects.

The covariance matrix that generates the jointness among the dependent variables is specified as follows (see Section 3.4):

$$
\begin{aligned}
& \operatorname{Var}(\breve{\boldsymbol{y}})=\breve{\mathbf{\Omega}}=\left[\begin{array}{llll}
1.000 & 0.600 & 0.600 & 0.000 \\
0.600 & 1.360 & 0.360 & 0.000 \\
0.600 & 0.360 & 1.000 & 0.200 \\
0.000 & 0.000 & 0.200 & 1.625
\end{array}\right] \\
& =\mathbf{L}_{\breve{\Omega}} \mathbf{L}_{\check{\Omega}}^{\prime}=\left[\begin{array}{llll}
1.00 & 0.00 & 0.00 & 0.00 \\
0.60 & 1.00 & 0.00 & 0.00 \\
0.60 & 0.00 & 0.80 & 0.00 \\
0.00 & 0.00 & 0.25 & 1.25
\end{array}\right]\left[\begin{array}{cccc}
1.00 & 0.60 & 0.60 & 0.00 \\
0.00 & 1.00 & 0.00 & 0.00 \\
0.00 & 0.00 & 0.80 & 0.25 \\
0.00 & 0.00 & 0.00 & 1.25
\end{array}\right]
\end{aligned}
$$


In the above $\breve{\mathbf{\Omega}}$ matrix, the first element is normalized (and fixed) to the value of 1, as is the third diagonal element (this third diagonal element corresponds to $\Sigma_{y^{*}}$ ). The sub-matrix of the first two columns and first two rows of $\breve{\mathbf{\Omega}}$ correspond to the matrix $\boldsymbol{\Sigma}_{\breve{u}^{*}}$ in Equation (4), which itself is the covariance matrix of the utility differentials of the second and third alternatives (with respect to the first alternative) in the nominal variable. In the simulation exercise, for convenience, we fix the covariance of the utility differentials in the nominal variable with the continuous variable to the value of zero. Then, there are five Cholesky matrix elements to be estimated in $\mathbf{L}_{\mathbf{\Omega}}$ $\left(l_{\check{\mathbf{\Omega}}_{1}}=0.6, l_{\check{\mathbf{\Omega}}_{2}}=1.0, l_{\breve{\Omega}_{3}}=0.6, l_{\check{\mathbf{\Omega}}_{4}}=0.25, l_{\breve{\Omega}_{5}}=1.25\right) .{ }^{5}$ Collectively, these elements, vertically stacked into a column vector, will be referred to as $l_{\check{\mathbf{\Omega}}}$.

The set-up above is used to develop the covariance matrix $\mathbf{\Omega}$ for the error vector $\boldsymbol{\tau}=\left(\varepsilon_{1}, \varepsilon_{2}, \varepsilon_{3}, \xi, \eta\right)^{\prime}$. The mean vector $\boldsymbol{V}=\left(V_{1}, V_{2}, V_{3}\right)^{\prime}$ for the utilities $\boldsymbol{U}=\left(U_{1}, U_{2}, U_{3}\right)^{\prime}$ in the nominal variable are also computed. Then, for each of the 2000 observations, a specific realization of the $\boldsymbol{\tau}$ vector is drawn from the multivariate normal distribution with mean $\boldsymbol{0}_{5}$ and covariance structure $\boldsymbol{\Omega}$. The realization corresponding to $\varepsilon=\left(\varepsilon_{1}, \varepsilon_{2}, \varepsilon_{3}\right)^{\prime}$ is added to the mean vector $\boldsymbol{V}$ to obtain the realization of the vector $\boldsymbol{U}$ for each observation. The alternative with the highest utility value is then picked, and identified as the chosen alternative for each observation. Next, the generated value for $y^{*}=\xi$ is translated into an observed count based on the computed threshold values (which include the dummy variables corresponding to the nominal variable). The value for the continuous variable $y$ is directly obtained from the realization for the error term $\eta$ after adding with the expected value computed for this dependent variable.

\footnotetext{
${ }^{5}$ In the covariance matrix $\breve{\boldsymbol{\Omega}}$, there are six parameters to be estimated, corresponding to two parameters in the covariance of the utility differentials of the MNP model (0.6 and 1.36), two parameters corresponding to the covariance between the two utility differentials in the MNP model with the count error term ( 0.6 and 0.36$)$, one parameter corresponding to the covariance between the count error term and the continuous model error term (0.2), and the one parameter corresponding to the variance of the continuous model error term (1.625). Thus, there should also be six parameters to estimate in the Cholseky decomposition too, and there are. It just so happens that one of those parameters to be estimated takes a value of 0 (this is in the third row and second column of $\mathbf{L}_{\breve{\Omega}}$ ). However, estimating this model leads to problems of assessing fit in our usual ways of computing percentage bias, the finite sample standard error as a percentage of the true value, etc. because of the division by zero (see Sections 3.2 and 3.3). So, in the simulation, we fix this parameter to zero, and estimate the other five parameters in the Cholesky matrix. This is just for convenience, and does not affect the parameter recovery analysis undertaken in the paper in any way.
} 
The above data generation process is undertaken 50 times with different realizations of the $\tau$ vector to generate 50 different data sets, each with 2000 observations. The MACML estimator is applied to each data set to estimate data specific values of $\left(\beta, \mu_{1}, \mu_{2}, \mu_{3}, \theta, \varphi_{1}, \varphi_{2}, \gamma, \boldsymbol{l}_{\bar{\Omega}}\right)$. A single random permutation is generated for each individual (the random permutation varies across individuals, but is the same across iterations for a given individual) to decompose the multivariate normal cumulative distribution (MVNCD) function into a product sequence of marginal and conditional probabilities (see Section 2.1 of Bhat, 2011). The estimator is applied to each dataset 10 times with different permutations to obtain the approximation error.

\subsection{Performance Evaluation}

The performance of the MACML inference approach in estimating the parameters of the proposed model and the corresponding standard errors is evaluated as follows:

(1) Estimate the MACML parameters for each data set and for each of 10 independent sets of permutations. Estimate the standard errors (s.e.) using the Godambe (sandwich) estimator.

(2) For each data set $s$, compute the mean estimate for each model parameter across the 10 random permutations used. Label this as MED, and then take the mean of the MED values across the data sets to obtain a mean estimate. Compute the absolute percentage (finite sample) bias (APB) of the estimator as:

$$
A P B=\left|\frac{\text { mean estimate }- \text { true value }}{\text { true value }}\right| \times 100
$$

(3) Compute the standard deviation of the MED values across the 50 data sets, and label this as the finite sample standard error or FSSE (essentially, this is the empirical standard error).

(4) For each data set, compute the mean s.e. for each model parameter across the 10 draws. Call this MSED, and then take the mean of the MSED values across the 50 data sets and label this as the asymptotic standard error or ASE (essentially this is the standard error of the distribution of the estimator as the sample size gets large).

(5) Next, to evaluate the accuracy of the asymptotic standard error formula as computed using the MACML inference approach for the finite sample size used, compute a relative efficiency (RE) value as:

$$
R E=\frac{\mathrm{ASE}}{\mathrm{FSEE}} \times 100
$$


(6) Compute the standard deviation of the parameter values around the MED parameter value for each data set, and take the mean of this standard deviation value across the data sets; label this as the approximation error (APERR).

\subsection{Simulation Results}

The results of the simulation experiments are presented in Table 1. The results indicate that the parameters in the formulation are recovered remarkably well by the estimation method. The absolute percentage bias (APB) is no more than 3\% for any parameter (see the column entitled "APB" under "Parameter Estimates"). The overall APB across all the parameters is a mere $0.8 \%$ (the bottom row of Table 1 under the column "APB"). Among all the non-covariance matrix parameters, the dispersion parameter of the underlying negative binomial distribution $(\theta)$ and the second parameter in the threshold parameterization $\left(\mu_{2}\right)$ are recovered least accurately with an APB value of $2.4 \%$ and $2.9 \%$ respectively. But these are still very good APB values. The reason for the relatively high APB value for the $\theta$ parameter is because this parameter appears very non-linearly in the model system of Equation (3), and through the $\psi_{l, n_{l}}$ threshold parameters. Among the Cholesky elements, the highest APB is observed for the $l_{\breve{\Omega}_{3}}$ element. This is the key parameter that introduces the endogeneity of the MNP model into the count model. Overall, the MACML method recovers the parameter extremely well, demonstrating the effectiveness of the MACML estimation approach.

The finite sample standard errors (FSSE) are small and are on an average about $10 \%$ of the true value of the parameters, indicating good empirical efficiency of the MACML estimator for the model. As a percentage of the true value, the FSSE is the least for the $\gamma$ parameter (1\%), which is the coefficient of the explanatory variable in the continuous dependent value variable. This is not surprising, since a continuous dependent variable provides much richer information than limiteddependent variables, and facilitates the estimation of the exogenous variable effects with less noise. The $\beta$ parameter of the MNP model also has a low FSSE at 5\% of its true value. This is the only parameter apart from the two covariance matrix elements that governs the MNP outcome (in the simulation exercise), and thus the full information in the MNP outcome goes to bear on estimating this parameter. The six structural parameters associated with the count outcome have a higher FSSE relative to their respective true values (an average FSSE of $14 \%$ of the true values). This may be attributable to the relatively higher number of parameters to be estimated in the count model, which 
naturally results in a little more noise in estimating each of the parameters. The Cholesky elements have FSSE values that are of the order of only $8 \%$ of the corresponding true values, indicating that these elements are also estimated with good precision.

The finite sample standard errors and the asymptotic standard errors obtained are very close, with the relative efficiency (RE) value between 0.89-1.10 for all parameters. The average RE value is 1.01 , indicating that the asymptotic formula is performing well in estimating the finite sample standard error. Further, as for the FSEE values, the ASE estimate, on average across all parameters, is also only $10 \%$ of the mean estimate, indicating very good efficiency even using the ASE estimate for the FSEE. Finally, the last column of Table 1 presents the approximation error (APERR) for each of the parameters, because of the use of different permutations. These entries indicate that the APERR is, on average, only 0.009 and the maximum is only 0.040 . More importantly, the approximation error (as a percentage of the FSEE or the ASE), averaged across all the parameters, is of the order of $13 \%$ of the sampling error. This is clear evidence that even a single permutation (per observation) of the approximation approach used to evaluate the MVNCD function provides adequate precision, in the sense that the convergent values are about the same for a given data set regardless of the permutation used for the decomposition of the multivariate probability expression.

\subsubsection{Effects of Ignoring the Joint Distribution of the Error Structures}

This section presents the results of the estimation when the endogeneity of the treatment variable on the count outcome is ignored. That is, we examine the effect of constraining $l_{\breve{\Omega}_{3}}$ to zero when the data actually reflects that the value is 0.6 . We expect that the net result would be that all the count model-related parameters would become biased (since the $l_{\check{\Omega}_{3}}$ parameter controls the amount of endogeneity in the MNP treatment effect on the count model). On the other hand, we do not expect additional bias in the MNP model, since it serves as the treatment in the simulation experiment, and so its parameters are consistently estimated even if the covariance in the treatment and the count outcome is ignored.

The simulation results for the restricted model (which we label as the "independent model") is presented in Table 2. For comparison purposes, we also present the results of the joint model proposed in the current paper. For the purpose of Table 2, we run only 50 estimations for each of the

independent and joint models, corresponding to each of the 50 data sets generated as per the 
experimental design of Section 3.1. That is, we use only one set of permutations per data set to evaluate the MVNCD functions and do not run ten estimation replications per data set with different sets of permutations. We do so because, as we presented in the earlier section, the approximation error in the parameters is negligible for any given data set. However, for each data set, we use the same set of permutations for the joint model and the independent model, so that we are able to appropriately compare the ability to recover parameters from the two models. In addition to an APB comparison between the joint model and the independent model, we also compare the performance of the two models using the adjusted composite log-likelihood ratio test (ADCLRT) value (see Pace et al., 2011 and Bhat, 2011 for more details on the ADCLRT statistic, which is the equivalent of the log-likelihood ratio test statistic when a composite marginal likelihood inference approach is used; this statistic has an approximate chi-squared asymptotic distribution). This statistic needs to be compared against the table chi-squared value with one degree of freedom, which is equal to 3.84 at the $5 \%$ level of significance. In this paper, we identify the number of times (corresponding to the 50 data sets) that the ADCLRT value rejects the independent model in favor of the joint model.

As can be observed from Table 2, the APB values are very substantially higher for all the count model-related parameters in the independent model. The overall APB across all parameters is $31.9 \%$ in the independent model relative to only $0.7 \%$ in the joint model (as discussed earlier, the joint model results in Table 2 are slightly different from those in Table 1, because we use only one set of permutations for the estimates in Table 2). The APB for the $\mu_{2}$ parameter is close to $200 \%$. Importantly, both the $\mu_{2}$ and $\mu_{3}$ parameters are substantially overestimated in the independent model, which is to be expected. Specifically, the true covariance matrix $\breve{\mathbf{\Omega}}$ shows a positive covariance of 0.6 between the utility differential of the second alternative (relative to the first) and the count outcome error, and a positive covariance of 0.36 between the utility differential of the third alternative (relative to the first) and the count outcome error. That is, unobserved factors that increase the utility of alternatives 2 and 3 (relative to alternative 1) also lead to an increase in the latent propensity driving the count outcome. When these covariances are forcibly suppressed, the model transfers the strong positive covariances to much higher positive (and biased) structural effects of the alternative 2 and alternative 3 dummy variables (with the first alternative being the base) in the count latent propensity, as is observed in the results. This exercise shows that accounting 
for endogeneity effects is not simply an esoteric econometric issue, but can have substantial implications for variable effects and subsequent policy analysis.

As expected, the $\beta$ parameter, and the $l_{\check{\Omega}_{1}}$ and $l_{\check{\Omega}_{2}}$ parameters, which correspond solely to the MNP model, continue to be estimated accurately. Also, the ADCLRT test toward the bottom of Table 2 clearly indicates that the joint model rejects the independent model in all the 50 data sets, further reinforcing the need to consider jointness in the MNP and count components when present.

\section{AN APPLICATION}

In this paper, we demonstrate the application of the proposed joint model by analyzing householdlevel decisions on residential location, motorized vehicle ownership, and activity-travel patterns.

\subsection{The Data}

The data source for this study is the 2009 National Household Travel Survey (NHTS) that collected complete out-of-home travel and activity information (as reported by respondents) for a sample of US households for a 24 hour survey period. In the current study, the survey subsample from the San Francisco-Oakland-San Jose, CA CMSA, encompassing 12 different counties including Alameda, Contra Costa, Marin, San Francisco, San Mateo, Santa Clara, San Benito, San Joaquin, Sonoma, Solano, Santa Cruz, and Napa, was extracted. This was done to limit the scope of the geographic region of analysis as well because the resulting region is diverse in terms of density. Each household's residential location was then assigned to one of the following density categories (housing units per square mile in the Census tract of the household's residence): (a) 0-99 households per square mile, (b) 100-499 households per square mile, (c) 500-1,999 households per square mile, and $(d) \geq 2,000$ households per square mile. These density categories were then used as the four discrete choice alternatives of a multinomial probit choice model. The number of motorized vehicles, one of the count dependent variables, is reported by households in the survey. All the rest of the dependent variables (number of tours made by motorized vehicles, number of tours made by non-motorized vehicles, and the natural logarithm of the average tour distance across motorized tours) are generated based on the travel diary filled in by the individuals of the household. In the rest of this paper, we will refer to the average tour distance across motorized tours more compactly as average motorized tour distance. 
The sample formation consisted of several steps. First, only households who responded to the survey on a weekday (Monday to Friday) were selected. Second, we eliminated households with individuals whose trip diary did not start or end at home. Third, records that contained incomplete information on individual, household, socioeconomic, and activity and travel characteristics of relevance to the current analysis were removed from the sample. Fourth, consistency checks were performed and records with missing or inconsistent data were eliminated. The final data sample used in the estimation included 2037 households that provided information on a host of demographic and travel variables of importance to this study.

\subsection{Dependent Variable Characteristics}

A tour is defined as a closed chain, with the beginning and ending of the tour being a specific base location. Only home-based tours and work-based tours are considered in this paper. If an individual travels from home to work in the morning, then stays at work until noon when she travels to a restaurant for lunch, next comes back to work for the entire afternoon and finally returns home in the evening, this is counted as two tours in the day; a home-based tour and a work-based tour. If in at least one leg of the tour, the individual uses a motorized mode of travel (car, bus, truck, van, SUV, motorcycle, taxicab, shuttle, ferry or train), the entire tour is considered to be made by a motorized vehicle (this is because tours can include short walk legs to get to the car or to get to the public transit station). The non-motorized modes are walk and bicycle, and a non-motorized tour corresponds to a tour in which all legs are pursued by walk and/or using a bicycle. For the continuous variable, we construct the natural logarithm of average motorized tour distance to avoid negative distance forecasts. ${ }^{6}$

Table 3 provides descriptive statistics for the three types of dependent variables used in the model. The top panel, associated with the nominal variable corresponding to household residential location, indicates that a small fraction of households (slightly more than 5\%) are located in the lowest density category, while nearly $50 \%$ of the households are located in the highest density category. The frequency distributions of the three count variables are presented in the bottom panel of the table. As expected, there are few households that have no cars or that make no motorized tours

\footnotetext{
${ }^{6}$ For completeness, we could have also constructed the average tour distance across non-motorized tours and used this as another continuous variable, but constructing (from the reported respondent data) the distances associated with non-motorized tours proved to be difficult because of the poor quality of data related to non-motorized tours.
} 
during the day, though there are quite a few households with zero non-motorized tours in the day. After introducing exogenous variables, flexibility terms $\left(\varphi_{l, j_{l}}\right)$ can be introduced as needed to accommodate the distribution of the counts (see Section 2.2). The average household values for the three count variables are 2.04 for motorized vehicle ownership, 2.79 for the number of daily motorized tours, and 0.55 for the number of non-motorized tours. The final dependent variable is the natural logarithm of the average motorized tour distance, which has an average value of 2.68 . The corresponding mean value for the motorized tour distance is 25.45 miles.

There are clear variations in the mean values for the count variables and the average motorized tour distance by residential density. For instance, the mean values of household motorized vehicle ownership are as follows for the last two density categories that capture about $82 \%$ of all households in the sample: 2.215 for the 500-1,999 households per square mile category and 1.845 for the highest density (greater than or equal to 2000 households per square mile category). The corresponding values for the number of motorized vehicle tours are 3.094 and 2.612, for the number of non-motorized tours are 0.511 and 0.629 , for average motorized tour distance are 27.92 and 21.66, and for implied VMT (product of the number of motorized tours and average motorized tour distance) are 85.52 and 58.46. Of course, these do not reflect the causal effects of residential density, because the differences may be attributable to the demographics and/or the attitudes/lifestyles of households residing in different locations. The purpose of the analytic model proposed in the paper is to account for these household characteristics, so that we may be able to isolate the "true" effects of residential density on activity-travel choices.

\subsection{Variable Specification and Model Formulation}

Five sets of independent variables were considered in the analysis: (1) family structure variables, including single person household, single parent household (one adult and at least one child 16 years old or younger), couple household (one male adult and one female adult), nuclear family household (one male adult, one female adult, and one or two children 16 years old or younger), and other households (primarily roommate and joint families; for ease, we will refer to these "other households" as "joint families"), (2) logarithm of household annual income, (3) household race and ethnicity, categorized as non-Hispanic Caucasian, African-American, Hispanic, and other (primarily Asian, but also including mixed race, pacific islander, and unidentified race; for ease, we will refer 
to these "other" households as "Asian" households"), (4) highest education attainment across individuals in the household (lower than Bachelor's degree, and Bachelor's degree or higher), and (5) Immigration status, including immigrant household (all members born outside the United States), non-immigrant household (all members born in the United States), and combination household (some members born in the United States, and others born outside the United States). The base alternatives for the categorical variables were as follows: Single person household (for family structure), "non-Hispanic Caucasian" race (for the household race and ethnicity variables), "lower than Bachelor's degree" (for education attainment), and "non-immigrant household" (for household immigration status).

In the analysis, we did not consider other variables such as housing type (i.e., whether the household lives in a duplex or townhouse or an apartment or a single family unit), housing tenure (owning or renting a home), number of drivers in a household, and household residence location in an urban or non-urban area, because of concerns that many of these variables themselves may be codetermined with the endogenous variables considered in the current analysis (this also suggests that the methodological framework proposed in this paper can be extended to include a few other endogenous variables in a larger integrated model, but which we leave for further research).

The exogenous variables were considered in the MNP utility specification, in the three count model threshold specifications, and in the log-linear mileage equation specification. The final variable specification was based on a systematic process of statistical significance testing, and combining variable effects if their impacts were not statistically different and if intuitive to do so. This search process was also informed by previous research and parsimony considerations. Simultaneously, a number of model structures with alternative structural relationships among the endogenous variables were compared against each other in terms of statistical measures of fit. In the end, after extensive testing, plausibility checks, and goodness-of-fit assessment, our results indicated that residential location structurally affects the number of vehicles and the (log) average motorized tour distance, and the number of vehicles affects the number of motorized tours, number of nonmotorized tours, and the $(\log )$ average motorized tour distance. However, our results also indicated statistically significant covariance terms among the error terms in the latent propensities underlying the observed outcome variables, indicating the presence of unobserved self-selection effects. That is, the recursive structural system does not mean that one can use a sequential modeling system; rather, 
the joint model system proposed in the paper is needed to capture the "bundling" of choices (see Section 1.3).

The MNP residential choice model is estimated with the highest density category $(\geq 2,000$ households per square mile) as the base alternative. For each of the three count dependent variables $(l=1,2,3)$, there are two parameter vectors $\left(\boldsymbol{\varphi}_{l}\right.$ and $\left.\boldsymbol{\mu}_{l}\right)$ and one scalar $\left(\theta_{l}\right)$ embedded in the threshold functions. Among these, the elements of the vector $\varphi_{l}$ provide flexibility to accommodate high or low probability masses for specific count outcomes that cannot be explained by the underlying parameterized Negative Binomial probabilities. In our estimations, we needed one flexibility term corresponding to $\varphi_{1,1}$ for the number of motorized vehicles count model (a value of 0.58 with a tstatistic of 10.68) and one flexibility term corresponding to $\varphi_{2,1}$ for the number of motorized tours model (a value of 0.72 with a t-statistic of 11.13). Also, the model specifications for these two count variables (the number of motorized vehicles and the number of motorized tours) collapsed to a Poisson generating process. In particular, the $\theta_{l}$ parameters for these two count variables $(l=1,2)$ became quite large in the estimations, and the resulting specifications could not be distinguished from corresponding Poisson-based latent variable specifications. However, the $\theta_{l}$ parameter clearly revealed the need for the more general negative binomial specification for the number of nonmotorized tours $(l=3)$. This parameter had a value of 0.908 , with a standard error of 0.105 .

\subsection{Model Estimation Results}

Table 4 provides the estimation results. We do not present the standard errors or t-statistics to reduce clutter. But unless otherwise noted, all the parameters in Table 4 are statistically different from zero at the $5 \%$ level of significance.

In the multinomial probit (MNP) model in the left panel of the table, if a '-' appears for a row variable in Table 4 corresponding to a column alternative (under the broad MNP residential choice model column), it implies that the corresponding row variable has no differential effect on the utilities of the lowest density category and the column alternative. Also, there is no intuitive interpretation of the constants in the MNP model because of the presence of continuous variables in the model. In the count models, the focus will be on the elements of the $\boldsymbol{\mu}_{l}$ vector $(l=1,2,3)$ embedded in the threshold functions, because the other parameters vectors $\left(\varphi_{l}\right.$ and $\left.\theta_{l}\right)$ have already 
been discussed in the previous section. The constant coefficient in the $\boldsymbol{\mu}_{\boldsymbol{l}}$ vector does not have any substantive interpretation. For the other variables, a positive coefficient in the $\boldsymbol{\mu}_{\boldsymbol{l}}$ vector shifts all the thresholds toward the left of the count propensity scale, which has the effect of reducing the probability of zero count (see CPB). On the other hand, a negative coefficient shifts all the thresholds toward the right of the count propensity scale, which has the effect of increasing the probability of zero count.

\subsubsection{Exogenous Variable Effects}

The effects of the many family structure variables in Table 4, in totality, indicate that single person households, single parent households, and nuclear family households are more likely than couple family households and joint family households to locate in higher density areas. Equivalently, couple family households and joint family households have a preference to locate in lower density areas than single individual households, single parent households, and nuclear family households. Earlier research (see Kim, 2011) does suggest that single adult and single parent households tend to locate themselves in denser neighborhoods, so that they are able to partake easily in social and related activity opportunities. The effects of the family structure variables on the other dependent variables (see the columns titled "Counts" and "Linear Regression") are rather intuitive (the parameters in the columns for the count variables are coefficients corresponding to the $\boldsymbol{\mu}_{\boldsymbol{l}}$ vector). In general, as the number of adults in a household increases (as reflected in the family structure variables), so do the number of motorized vehicles in the household and the number of motorized tours made by the household. These indications are consistent with expectations and with the now vast literature on auto ownership modeling (see, for example, Potoglou and Susilo, 2008 and Ma and Srinivasan, 2010). Also, couple households make more non-motorized tours than do single person households, while non-single and non-couple households have a higher propensity to make nonmotorized tours than do single person and couple households. These results most likely reflect joint activity participation in recreation activities (such as walking around the neighborhood or walking to a park), which tend to increase in the presence of multiple adults and children in the household (see Lee et al., 2007). Overall, among all households, single person households are the least likely to own vehicles, and make motorized and non-motorized tours. According to the 2009-2011 American Community Survey three-year estimates, as in our sample, a third of the single person households in 
the SFO area are elderly (age 65 or more), which is consistent with the result just mentioned. Finally, in the category of family structure variables, the higher average tour distance in couple households and other relative to other types of households perhaps reflect joint travel episodes that also serve as time alone together for the couple.

Households with high income tend to stay away from highly dense areas, perhaps a reflection of being able to afford large single-family homes in suburban locations. This residential location pattern based on income has been observed in large cities (see Cao and Fan, 2012). Income also has the expected positive impacts on the number of motorized vehicles owned, the number of motorized vehicle tours, and the average distance per tour.

Interestingly, we did not find much differences in residential location based on race, except for a higher tendency among those of Asian race to locate in the highest density $(>=2000$ households per square mile) neighborhoods. This finding is quite different from some other studies that show substantial differences in residential location preferences (in terms of neighborhood density) between Caucasian and non-Caucasian households (for example, in Giuliano, 2003 and Cao and Fan, 2012). The relative absence of race impacts in our study is perhaps in part because we have controlled separately for immigrant status effects, while many earlier studies have not. Indeed, when the immigrant status effects were removed in our model, the race effects on residential location became statistically significant. In terms of race effects on other dependent variables, households of Hispanic race make fewer motorized tours, while households of Asian race make fewer nonmotorized tours. A few earlier studies (see Allen et al., 2007 and Dogra et al., 2010) in the public health field also have observed that Asian households tend to be less physically active in terms of non-motorized recreation pursuits (such as walking and bicycling around the neighborhood).

Households with high education levels, and households with one or more immigrants, favor residential locations in non-low density neighborhoods. The former result may reflect a desire among households with highly educated individuals to locate in denser neighborhoods with "high culture" arts/recreation activity opportunities, while the clustering of immigrant households in relatively high density neighborhoods is consistent with the large body of literature on the subject (see, for example, Wilson and Singer, 2011 and Bhat et al., 2013 for two recent studies). 


\subsubsection{Structural Dependence Effects}

The household location density categories are considered to be endogenous in the proposed joint model of this paper. Thus, the effects of the density on Table 4 are "cleansed" of the unobserved factors that generate a correlation between the propensity of locating to a specific residential density category and the three count propensities (see next section). The results indicate, as expected, that households are less likely to own zero motorized vehicles (more likely to own motorized vehicles) if they are located in low density areas (relative to being located in the highest density area). Interestingly though, there is no direct structural effect of residential location on the number of motorized and non-motorized tours. However, there is a direct structural effect of residential location on the natural logarithm of average motorized tour distance. The results indicate that households residing in progressively less dense neighborhoods make, in general, longer distance motorized tours, a finding also observed by Maat and Timmermans (2006). In addition, Table 4 reveals the positive structural effects of the number of vehicles on the number of motorized tours and motorized tour length, and the negative structural effect of the number of vehicles on the number of nonmotorized tours. These findings are consistent with the results from earlier studies such as Cao et al. (2009b).

\subsubsection{Covariance Matrix}

Many different specifications were considered for the covariance matrix. In the MNP model, a general specification was considered for the covariance matrix $\breve{\Lambda}_{1}$ of the error differences. But, in our empirical context, we could not reject the null hypothesis that this matrix has ones in its diagonals and 0.5 entries in its off-diagonals. This, of course, is equivalent to an independent and identical distribution specification for the original error terms (that is, the $\boldsymbol{\Lambda}$ covariance matrix of the original error terms turns out to be an identity matrix multiplied by 0.5 ). However, this result is specific to the current empirical context. In general, one needs to specify the more general model proposed in this paper before testing for more restrictive variants.

Table 5 presents the covariance matrix estimates, which indicate that the elements corresponding to the covariance between the utility differential of the third density category (with respect to the highest density category) and the number of vehicles is positive and statistically significant (see the entry ' 0.073 ' in the row entitled "number of vehicles and the column entitled 
"500-1999"). The one corresponding to the second density category (with respect to the highest density segment) is also statistically significant, although smaller in magnitude (this is the entry ' 0.052 '). The implication is that unobserved factors that increase the preference for locating in lower density neighborhoods (specifically, neighborhoods with a density of 100-499 or 500-1999 households per square mile) relative to very high density neighborhoods (2000 or more households per square mile) also increase the propensity to own motorized vehicles. This may represent the effects of such factors as auto inclination and not being very environmentally conscious. Alternatively, those who choose to reside in high density, neo-urbanist type neighborhoods appear to do so because of lifestyle choices that intrinsically are non-auto oriented. Of course, alternative explanations are also possible (precisely because the dependence is due to intrinsically unobserved factors). But the important point is that, if not controlled for, the positive covariance gets comingled with the true structural effect of high density on motorized vehicle ownership, inappropriately increasing the positive effect of relatively low density residence on motorized vehicle ownership. That is, the attribution to neo-urbanist neighborhoods (toward lower motorized vehicle ownership levels) gets exaggerated when residential self-selection effects are not considered. Indeed, we found this to be the case when we estimated a model that ignores the covariance across the dependent variables. The coefficient of the density category dummy variables increased in magnitude from 0.127 to 0.158 for the second density category (100-499 households per square mile) and from 0.088 to 0.131 for the third density category (500-1999 households per square mile).

Table 5 does not indicate statistically significant covariance elements between the MNP utility differences and the propensities underlying the counts of motorized tours and non-motorized tours. However, there are statistically significant covariance elements between the error terms of the different counts. The covariance between the number of vehicles and the number of motorized tours is positive and the covariance between the number of vehicles and the number of non-motorized tours is negative (see the 0.176 and -0.079 entries in the column labeled "number of motorized vehicles" in Table 5). That is, there are common unobserved household factors (such as say being auto-inclined) that simultaneously increase the propensity to own motorized vehicles and make motorized tours, while these same factors appear to have opposite effects on the propensity to own motorized vehicles and make non-motorized tours. Again, if we estimate a new model ignoring the covariance between the dependent variables, we obtain larger coefficients (in terms of magnitude) for the effect of number of vehicles on the number of motorized tours (coefficient changes from 
0.053 to 0.072 ) and on the number of non-motorized tours (coefficient changes from - 0.163 to 0.199). This shows that there are self-selection effects based on motorized vehicle ownership levels too on the number of motorized and non-motorized tours, reinforcing the point made in the introductory section that self-selection may not be confined to residential location decisions but may permeate through other decisions too in the structural chain of effects.

Finally, there are no significant covariance effects between the MNP /count propensity error terms and the error term in the log-linear model for the average motorized tour distance.

\subsubsection{Composite Log-Likelihood at Convergence}

The composite log-likelihood value for the joint model (with 49 parameters) is -35035.4 , while the corresponding value for the independent model (with 44 parameters) is $-35,074.3$. The two models may be compared using the adjusted composite likelihood ratio test (ADCLRT) statistic that is approximately chi-squared distributed (the ADCLRT statistic is similar to the likelihood ratio test statistic used in ordinary maximum likelihood estimation, though its construction is not as simple as the likelihood ratio statistic; see Bhat (2011) for a detailed discussion). The ADCLRT statistic value is 82.3 , which is larger than the chi-squared table value with 5 degrees of freedom at any reasonable level of significance. This result clearly illustrates the superior data fit offered by the joint model.

\subsection{Procedure for Treatment Effects Based on Residential Choice}

The estimation results can be used to assess the impact of residential location choice (the "treatment") on all the other dependent variables (the "outcomes"). This is helpful to obtain insights regarding whether, and how much, neo-urbanist design measures impact travel-related behaviors. An important measure to do so is the Average Treatment Effect (ATE) (see Heckman and Vytlacil, 2000 and Heckman et al., 2001).

In the context of motorized vehicle ownership, the ATE measure provides the expected difference in motorized vehicle ownership for a random household if it were located in a specific density configuration $i$ as opposed to another density configuration $k \neq i$. The measure is estimated as follows:

$$
\hat{\operatorname{ATE}}_{i k}=\frac{1}{Q} \sum_{q=1}^{Q}\left(\sum_{j_{1}=0}^{\infty} j_{1} \cdot\left[P\left(y_{q 1}=j_{1} \mid a_{q i}=1\right)-P\left(y_{q 1}=j_{1} \mid a_{q k}=1\right)\right]\right)
$$


where $a_{q i}$ is the dummy variable for the density category $i$ for the household $q$, and $y_{q 1}$ stands for motorized vehicle ownership with an index $j_{1}\left(j_{1}=0,1,2, \ldots \infty\right)$ (the subscript ' 1 ' indicates that motorized vehicle ownership is the first count variable in the model system). Although the summation in the equation above extends until infinity, we consider counts only up to $j_{1}=11$, which is the maximum motorized vehicle ownership level observed in the dataset. This should not affect the computations because the probabilities associated with higher motorized vehicle ownership levels are very close to zero.

The analyst can compute the ATE measures for all the pairwise combinations of residential density category relocations. Here, we focus on the case when a household in the penultimate high density neighborhood (500-1999 households per square mile) is transplanted to the highest density neighborhood ( $>=2000$ households per square mile). For ease in discussion, in the rest of this section, we will refer to the former neighborhood type as a low density neighborhood, and the latter neighborhood type as a high density neighborhood.

The analyst can also compute the ATE for the number of motorized and non-motorized tours based on residential location. Since the numbers of motorized and non-motorized tours are not structurally dependent, the computation becomes a little easier. So, the ATE expression corresponding to the number of motorized tours is:

$$
\hat{\mathrm{ATE}}_{i k}=\frac{1}{Q} \sum_{q=1}^{Q}\left(\sum_{j_{1}=0}^{11} j_{1} \cdot\left(\sum_{j_{2}=0}^{15} j_{2}\left[P\left(y_{q 1}=j_{1}, y_{q 2}=j_{2} \mid a_{q i}=1\right)-P\left(y_{q 1}=j_{1}, y_{q 2}=j_{2} \mid a_{q k}=1\right)\right]\right)\right) \text {. }
$$

The summation is being taken up to a count of 15 for the number of motorized tours because this was the maximum count in the estimation data. A similar approach may be taken for the ATE of the number of non-motorized tours. Next, for the ATE for the average motorized tour distance variable, we compute the expected values of the number of vehicles and tours and then substitute these in the linear regression expression for each household, obtain the expected value of the logarithm of motorized tour distance, and translate this to the expected value of motorized tour distance (doing so entails taking the exponential of the sum of the expected logarithm value and half the variance of the error term in the log-linear regression model). Finally, using the same approach as just discussed, one can also compute an ATE for vehicle miles of travel (VMT), since VMT is the product of the number of motorized tours and the average tour distance. The standard errors of the ATE measures for the many variables are obtained using bootstraps from the sampling distributions of the estimated 
parameters, but are suppressed here to focus the presentation and reduce clutter. Suffice it to say that all the ATE measures (from both the joint and the independent models) were statistically significant at the $5 \%$ level of significance.

Table 6 provides the ATE values for each of the five attributes for the joint model proposed here and the independent model that ignores all forms of self-selection. The first row in the first column under the "Joint Model" heading indicates that a random household that is shifted from the low density category location to the high density category location is, on an average, likely to reduce its motorized vehicle ownership level by 0.189 vehicles. Equivalently, if 100 random households are relocated from the low density neighborhood to the high density neighborhood, the results indicate a reduction in motorized vehicle ownership by about 19 vehicles. On the other hand, the independent model predicts a reduction of 0.261 vehicles. That is, if 100 random households are relocated from the low density neighborhood to the high density neighborhood, the independent model projects a reduction in motorized vehicle ownership by about 26 vehicles. The exaggeration in the reduction in motorized vehicle ownership based on the independent model (because of the change in residence from the low density to the high density neighborhood) is readily apparent, and is a reflection of unobserved residential self-selection effects not being controlled for. One can quantity the magnitude of the "true" effect and the spurious residential self-selection effect, because the independent model comingles both of these effects, while the joint model estimates the "true" effect. The last two columns of Table 6 indicate that unobserved self-selection effects are estimated to constitute about $28 \%$ of the difference in the number of motorized vehicles between low density and high density households, while "true" built environment effects constitute the remaining $72 \%$ of the difference.

The other columns of the table may be similarly interpreted. The results are consistent with the discussion in Section 4.4.3. Overall, the results show that, if self-selection effects are ignored, the result is an overestimation in the reduction in motorized vehicle ownership because of residing in high density neighborhoods. There is also an overestimation in the reduction in the number of motorized vehicle tours, and an overestimate in the increase in the number of non-motorized tours. In terms of VMT, the joint model predicts a reduction by 11.43 miles if a random household is moved from a low density neighborhood to a high density neighborhood, while the independent model predicts a much more optimistic (and inappropriate) reduction by almost 16 miles. In terms of order of magnitude effects relative to the average VMT ( $=72.2$ miles) across all households, the joint 
model predicts a VMT reduction of $15.8 \%$ due to moving a random household from a low density neighborhood to a high density neighborhood, while the independent model predicts a VMT reduction of $22.0 \%$ for the same move. To summarize, the results do show that density has important "true" effects on activity-travel behavior, but that these effects are exaggerated when self-selection is ignored.

\section{CONCLUSIONS}

This paper formulates a multidimensional choice model system that is capable of handling multiple nominal variables, multiple count dependent variables, and multiple continuous dependent variables. The system takes the form of a treatment-outcome selection system with multiple treatments and multiple outcome variables. The Maximum Approximate Composite Marginal Likelihood (MACML) approach proposed by Bhat (2011) is proposed in estimation, which, in a relatively simple and practical manner, provides a way out to estimate large multi-dimensional choice model systems. To our knowledge this is the first such sample selection formulation and application in the econometrics literature. A simulation experiment is undertaken to evaluate the ability of the MACML method to recover the model parameters in such integrated systems, as well as to assess the ability of the asymptotic standard errors from the analytic procedure to provide an estimate of the finite sample errors for the typical sample sizes employed in estimation. These experiments show that our estimation approach recovers the underlying parameters very well and is efficient from an econometric perspective.

The parametric model system proposed in the paper is applied to an analysis of householdlevel decisions on residential location, motorized vehicle ownership, the number of daily motorized tours, the number of daily non-motorized tours, and the average distance for the motorized tours. The empirical analysis uses the NHTS 2009 data from the San Francisco Bay area. Model estimation results show that the choice dimensions considered in this paper are inter-related, both through direct observed structural relationships and through correlations across unobserved factors (error terms) affecting multiple choice dimensions. The significant presence of self-selection effects (endogeneity) suggests that modeling the various choice processes in an independent sequence of models is not reflective of the true relationships that exist across these choice dimensions, as also reinforced through the computation of treatment effects in the paper. These treatment effects also emphasize that accounting for residential and other self-selection effects are not simply esoteric econometric 
pursuits, but can have important implications for land-use policy measures that focus on neourbanist design. Importantly, our results indicate that not accommodating self-selection effects may lead to an overestimation in the projected reduction in motorized travel attributed to land-use densification measures.

To summarize, this paper proposes and demonstrates the use of an integrated framework to model multiple variables of multiple types. The proposed model can be applied to a wide variety of contexts in different disciplines. Future efforts need to continue to undertake simulation experiments to evaluate the performance of the MACML approach for estimating large-scale integrated model systems. From an empirical perspective, the model in this paper can be extended to include additional count variables related to the number of out-of-home episodes by purpose.

\section{ACKNOWLEDGEMENTS}

Lisa Macias helped with typesetting and formatting this document. 


\section{REFERENCES}

Allen, M.L., Elliott, M.N., Morales, L.S., Diamant, A.L., Hambarsoomian, K., and Schuster, M.A. (2007). Adolescent participation in preventive health behaviors, physical activity, and nutrition: differences across immigrant generations for Asians and Latinos compared with Whites. American Journal of Public Health, 97(2), 337-343.

Bamberg, S. (2013). Applying the stage model of self-regulated behavioral change in a car use reduction intervention. Journal of Environmental Psychology, 33, 68-75

Bhat, C.R. (2011) The maximum approximate composite marginal likelihood (MACML) estimation of multinomial probit-based unordered response choice models. Transportation Research Part B, 45(7), 923-939.

Bhat, C.R., and Guo, J.Y. (2007) A comprehensive analysis of built environment characteristics on household residential choice and auto ownership levels. Transportation Research Part B, 41(5) 506-526.

Bhat, C.R., and Eluru, N. (2009). A copula-based approach to accommodate residential selfselection effects in travel behavior modeling. Transportation Research Part B, Vol. 43(7), 749-765.

Bhat, C.R., and Sidharthan, R. (2012). A new approach to specify and estimate non-normally mixed multinomial probit models. Transportation Research Part B, 46, 817-833.

Bhat, C.R., Sen, S., and Eluru, N. (2009). The impact of demographics, built environment attributes, vehicle characteristics, and gasoline prices on household vehicle holdings and use. Transportation Research Part B, 43(1), 1-18.

Bhat, C.R., Paleti, R., Pendyala, R.M., Lorenzini, K., and Konduri, K.C. (2013), Accommodating immigration status and self-selection effects in a joint model of household auto ownership and residential location choice. Transportation Research Record: Journal of the Transportation Research Board, forthcoming.

Brownstone, D., and Golob, T., 2009. The impact of residential density on vehicle usage and energy consumption. Journal of Urban Economics, 65, 91-98.

Cao, X., and Fan, Y. (2012). Exploring the influences of density on travel behavior using propensity score matching. Environment and Planning B, 39(3), 459.

Cao, X., Mokhtarian, P.L., and Handy, S.L. (2009a). Examining the impacts of residential selfselection on travel behavior: a focus on empirical findings, Transport Reviews, 29(3), 359395.

Cao, X., Mokhtarian, P.L., and Handy, S.L. (2009b). The relationship between built environment and nonwork travel: A case study of Northern California, Transportation Research Part A, 43(5), 548-559.

Cao, X. and Fan, Y. (2012). Exploring the influences of density on travel behavior using propensity score matching, Environment and Planning B, 39, 459-470. 
Castro, M., Paleti, R., and Bhat, C.R. (2012) A latent variable representation of count data models to accommodate spatial and temporal dependence: application to predicting crash frequency at intersections. Transportation Research Part B, 46(1), 253-272.

Chatman, D.G. (2009). Residential choice, the built environment, and nonwork travel: evidence using new data and methods. Environment and Planning A, 41, 1072-1089.

Cox, D.R. and Reid, N. (2004). A note on pseudolikelihood constructed from marginal densities. Biometrika, 91(3), 729-737.

de Abreu e Silva, J., Morency, C., and Goulias, K.G. (2012) Using structural equations modeling to unravel the influence of land use patterns on travel behavior of workers in Montreal, Transportation Research Part A, 46(8), 1252-1264.

Dogra, S., Meisner, B.A., and Ardern, C.I. (2010). Variation in mode of physical activity by ethnicity and time since immigration: a cross-sectional analysis. International Journal of Behavioral Nutrition and Physical Activity, 7(1), 75.

Eluru, N., Pinjari, A.R., Pendyala, R.M., and Bhat, C.R. (2010). An econometric multi-dimensional choice model of activity-travel behavior. Transportation Letters: The International Journal of Transportation Research, 2(4), 217-230.

Giuliano, G. (2003). Travel, location and race/ethnicity. Transportation Research Part A, 37, 351372.

Godambe, V.P. (1960). An optimum property of regular maximum likelihood estimation. The Annals of Mathematical Statistics, 31(4), 1208-1211.

Handy, S. and Krizek, K. (2012). The role of travel behavior research in reducing carbon footprint: A US perspective. In Pendyala, R.M. and Bhat C.R. (eds), Travel Behaviour Research in an Evolving World: Selected Papers from the $12^{\text {th }}$ International Conference on Travel Behavior Research, Lulu.com Publishers.

Heckman, J.J. and Vytlacil, E. (2000). The relationship between treatment parameters within a latent variable framework. Economics Letters, 66(1), 33-39.

Heckman, J.J., Tobias, J.L., and Vytlacil, E. (2001). Four parameters of interest in the evaluation of social programs. Southern Economic Journal, 68(2), 210-223.

Keane, M.P. (1992). A note on identification in the multinomial probit model. Journal of Business \& Economic Statistics, 10(2), 193-200.

Kim, S. (2011). Intra-regional residential movement of the elderly: testing a suburban-to-urban migration hypothesis. The Annals of Regional Science, 46(1), 1-17.

Kim, J. and Brownstone, D. (2012). The impact of residential density on vehicle usage and fuel consumption: evidence from national samples. Department of Economics, University of California, Irvine, http://sites.uci.edu/jinwonkim/files/2012/05/paper_0423.pdf, accessed April 7, 2013. 
Lee, Y., Hickman, M. and Washington, S. (2007). Household type structure, time-use pattern, and trip-chaining behavior. Transportation Research Part A, 41(10), 1004-1020.

Levine, J. (2006). Zoned out: Regulation, markets, and choices in transportation and metropolitan land use. Resources for the Future, Washington, D.C.

Lindsay, B.G. (1988) Composite likelihood methods. Contemporary Mathematics, 80, 221-239.

Lindsay, B.G., Yi, G.Y. and Sun, J. (2011). Issues and strategies in the selection of composite likelihoods. Statistica Sinica, 21(1), 71-105.

Ma, L., and Srinivasan, S. (2010). Impact of individuals' immigrant status on household auto ownership. Transportation Research Record: Journal of the Transportation Research Board, 2156, 36-46.

Maat, K., and Timmermans, H. (2006). Influence of land use on tour complexity: a Dutch case. Transportation Research Record: Journal of the Transportation Research Board, 1977, 234241.

Maddala, G.S. (1983) Limited-Dependent and Qualitative Variables in Econometrics, Cambridge University Press, Cambridge, UK.

Mokhtarian, P.L., and Cao, X. (2008) Examining the impacts of residential self-selection on travel behavior: A focus on methodologies. Transportation Research Part B, 42(3), 204-228.

Molenberghs, G., Verbeke, G., 2005. Models for Discrete Longitudinal Data. Springer Series in Statistics. Springer Science + Business Media, Inc., New York.

Munkin, M.K., Trivedi, P.K. (2008). Bayesian analysis of the ordered probit model with endogenous selection. Journal of Econometrics, 143(2), 334-348.

Pace, L., Salvan, A., Sartori, N., 2011. Adjusting composite likelihood ratio statistics. Statistica Sinica 21(1), 129-148.

Paleti, R., Bhat, C.R., and Pendyala, R.M. (2013), An integrated model of residential location, work location, vehicle ownership, and commute tour characteristics. Transportation Research Record: Journal of the Transportation Research Board, forthcoming.

Pinjari, A.R., Pendyala, R.M., Bhat, C.R., and Waddell, P.A. (2011), Modeling the choice continuum: an integrated model of residential location, auto ownership, bicycle ownership, and commute tour mode choice decisions. Transportation, 38(6), 933-958.

Potoglou, D., and Susilo, Y.O. (2008). Comparison of vehicle-ownership models. Transportation Research Record: Journal of the Transportation Research Board, 2076, 97-105.

Puhani, P.A. (2000). The Heckman correction for sample selection and its critique. Journal of Economic Surveys, 14(1), 53-68. 
Salon, D., Boarnet, M.G., Handy, S., Spears, S., Tal, G. (2012). How do local actions affect VMT? A critical review of the empirical evidence. Transportation Research Part D, 17(7), 495508.

Spears, S., Houston, D., and Boarnet, M. (2012). Illuminating the unseen in neighborhood travel: A framework for examining the influence of attitudes, norms, and perceptions on travel behavior, Presented at the ACSP $53^{\text {rd }}$ Annual Conference, Cincinatti, Ohio.

Sperry, B.R. Burris, M.W., and Dumbaugh E. (2012) A case study of induced trips at mixed-use developments. Environment and Planning B, 39(4), 698-712.

Transportation Research Board (2009) Driving and the Built Environment: The effects of compact development on motorized travel, energy use, and Co2 emissions. Special Report 298, The National Academies Press, Washington, D.C.

Van Acker, V., Mokhtarian, P.L., and Witlox, F. (2011). Going soft: on how subjective variables explain modal choices for leisure travel. European Journal Of Transport And Infrastructure Research, 11(2), 115-146.

Van Acker, V., Boussauw, K., Derudder, B., and Witlox, F. (2012). The causal influence of the built environment questioned: self-selection, underlying attributes, and feedback mechanisms, Proceedings of the $21^{\text {st }}$ Annual Meeting of the Transportation Research Board, Washington, D.C.

Vance, C., and R. Hedel (2007). The impact of urban form on automobile travel: disentangling causation from correlation. Transportation, 34(5), 575-588.

Varin, C., Reid, N., and Firth, D. (2011) An overview of composite marginal likelihoods. Statistica Sinica, 21(1), 5-42.

Wilson, J., and Singer, A. (2011). Immigrants in 2010 Metropolitan America: A decade of change. Metropolitan Policy Program, Brookings Institution.

$\mathrm{Xu}, \mathrm{X}$. , and Reid, N. (2011). On the robustness of maximum composite likelihood estimate. Journal of Statistical Planning and Inference, 141(9), 3047-3054.

Yi, G.Y., Zeng, L., and Cook, R.J. (2011). A robust pairwise likelihood method for incomplete longitudinal binary data arising in clusters. Canadian Journal of Statistics, 39(1), 34-51.

Zhang, L., Hing, J., Nasri, A., and Shen, Q. (2012) How built environment affects travel behavior: A comparative analysis of the connections between land-use and vehicle miles traveled in US cities. The Journal of Transport and Land Use, 5(3), 40-52.

Zhao, Y., and Joe, H. (2005). Composite likelihood estimation in multivariate data analysis. The Canadian Journal of Statistics, 33(3), 335-356. 


\section{LIST OF TABLES}

Table 1. Simulation Results for 50 Datasets of 2000 Observations Each

Table 2. Effect of Ignoring Endogenous Effects

Table 3. Sample Characteristics

Table 4. Model Estimation Result

Table 5. Model Estimation Result - Covariance Matrix

Table 6. Treatment Effects Corresponding to Transplanting a Random Household from a 5001999 HHs per Sq. Mile Density Neighborhood to the Highest Density Neighborhood 
Table 1. Simulation Results for 50 Datasets of 2000 Observations Each

\begin{tabular}{|c|c|c|c|c|c|c|c|c|}
\hline \multirow{2}{*}{ Parameter } & \multirow{2}{*}{$\begin{array}{c}\text { Component } \\
\text { of }\end{array}$} & \multicolumn{3}{|c|}{ Parameter Estimates } & \multicolumn{4}{|c|}{ Standard Error Estimates } \\
\hline & & True & $\begin{array}{c}\text { Mean } \\
\text { Estimate }\end{array}$ & APB & FSSE & ASE & RE & APERR \\
\hline$\beta$ & MNP & -1.000 & -1.002 & $0.2 \%$ & 0.050 & 0.046 & 0.933 & 0.005 \\
\hline$\mu_{1}$ & Count & 0.500 & 0.500 & $0.1 \%$ & 0.027 & 0.029 & 1.069 & 0.003 \\
\hline$\mu_{2}$ & Count & 0.250 & 0.257 & $2.9 \%$ & 0.067 & 0.066 & 0.986 & 0.011 \\
\hline$\mu_{3}$ & Count & 0.500 & 0.501 & $0.3 \%$ & 0.065 & 0.066 & 1.016 & 0.009 \\
\hline$\theta$ & Count & 2.000 & 2.047 & $2.4 \%$ & 0.258 & 0.272 & 1.054 & 0.040 \\
\hline$\varphi_{1}$ & Count & 0.300 & 0.301 & $0.4 \%$ & 0.044 & 0.041 & 0.933 & 0.005 \\
\hline$\varphi_{2}$ & Count & 0.600 & 0.600 & $0.0 \%$ & 0.070 & 0.070 & 0.999 & 0.010 \\
\hline$\gamma$ & Continuous & 2.000 & 2.005 & $0.3 \%$ & 0.029 & 0.028 & 0.971 & 0.001 \\
\hline$l_{\breve{\Omega}_{1}}$ & Covariance & 0.600 & 0.604 & $0.7 \%$ & 0.062 & 0.066 & 1.058 & 0.009 \\
\hline$l_{\breve{\Omega}_{2}}$ & Covariance & 1.000 & 1.003 & $0.3 \%$ & 0.074 & 0.066 & 0.895 & 0.008 \\
\hline$l_{\check{\mathbf{\Omega}}_{3}}$ & Covariance & 0.600 & 0.585 & $2.5 \%$ & 0.038 & 0.039 & 1.027 & 0.013 \\
\hline$l_{\check{\mathbf{\Omega} 4}_{4}}$ & Covariance & 0.250 & 0.249 & $0.4 \%$ & 0.035 & 0.037 & 1.045 & 0.004 \\
\hline$l_{\check{\mathbf{\Omega} 5}}$ & Covariance & 1.250 & 1.250 & $0.0 \%$ & 0.019 & 0.020 & 1.096 & 0.001 \\
\hline \multicolumn{2}{|c|}{ Across all Parameters } & & & $0.8 \%$ & 0.065 & 0.065 & 1.006 & 0.009 \\
\hline
\end{tabular}


Table 2. Effect of Ignoring Endogenous Effects

\begin{tabular}{|c|c|c|c|c|c|c|}
\hline \multirow{2}{*}{ Parameter } & \multirow{2}{*}{ Component of } & \multirow{2}{*}{ True } & \multicolumn{2}{|c|}{ Joint } & \multicolumn{2}{|c|}{ Independent } \\
\hline & & & $\begin{array}{c}\text { Mean } \\
\text { Estimate }\end{array}$ & APB & $\begin{array}{c}\text { Mean } \\
\text { Estimate }\end{array}$ & APB \\
\hline$\beta$ & MNP & -1.000 & -1.002 & $0.2 \%$ & -0.999 & $0.1 \%$ \\
\hline$\mu_{1}$ & Count & 0.500 & 0.500 & $0.1 \%$ & 0.527 & $5.4 \%$ \\
\hline$\mu_{2}$ & Count & 0.250 & 0.258 & $3.2 \%$ & 0.744 & $197.6 \%$ \\
\hline$\mu_{3}$ & Count & 0.500 & 0.501 & $0.2 \%$ & 0.727 & $45.3 \%$ \\
\hline$\theta$ & Count & 2.000 & 2.041 & $2.1 \%$ & 1.518 & $24.1 \%$ \\
\hline$\varphi_{1}$ & Count & 0.300 & 0.301 & $0.2 \%$ & 0.447 & $49.0 \%$ \\
\hline$\varphi_{2}$ & Count & 0.600 & 0.600 & $0.1 \%$ & 0.858 & $42.9 \%$ \\
\hline$\gamma$ & Continuous & 2.000 & 2.005 & $0.3 \%$ & 2.006 & $0.3 \%$ \\
\hline$l_{\breve{\Omega}_{1}}$ & Covariance & 0.600 & 0.608 & $1.3 \%$ & 0.600 & $0.1 \%$ \\
\hline$l_{\check{\mathbf{\Omega} 2}_{2}}$ & Covariance & 1.000 & 1.004 & $0.4 \%$ & 0.999 & $0.1 \%$ \\
\hline$l_{\breve{\Omega}_{4}}$ & Covariance & 0.250 & 0.249 & $0.3 \%$ & 0.208 & $16.8 \%$ \\
\hline$l_{\mathbf{\Omega}_{5}}$ & Covariance & 1.250 & 1.250 & $0.0 \%$ & 1.258 & $0.6 \%$ \\
\hline \multicolumn{3}{|c|}{ Overall mean value across parameters } & & $0.7 \%$ & & $31.9 \%$ \\
\hline \multicolumn{3}{|c|}{$\begin{array}{c}\text { Mean log composite marginal likelihood } \\
\text { at convergence }\end{array}$} & \multicolumn{2}{|c|}{-7145.78} & \multicolumn{2}{|c|}{-7211.02} \\
\hline \multicolumn{3}{|c|}{$\begin{array}{l}\text { Number of times the adjusted composite } \\
\text { likelihood ratio test (ADCLRT) statistic } \\
\text { favors the Joint model }\end{array}$} & \multicolumn{4}{|c|}{$\begin{array}{l}\text { All fifty times when compared with the value of } \\
\left.\chi_{1,0.95}^{2}=3.84 \text { (mean ADCLRT statistic is } 65.2\right)\end{array}$} \\
\hline
\end{tabular}


Table 3. Sample Characteristics

\begin{tabular}{|c|c|c|c|c|c|c|}
\hline \multicolumn{7}{|c|}{ Descriptive statistics } \\
\hline \multicolumn{7}{|c|}{ Dependent variables: MNP Variables } \\
\hline \multicolumn{3}{|c|}{ Location density [HHs per sq. mile] } & \multicolumn{4}{|c|}{ Number of observations ( $\%)$} \\
\hline \multicolumn{3}{|l|}{$0-99$} & \multicolumn{4}{|c|}{$108(5.30)$} \\
\hline \multicolumn{3}{|l|}{$100-499$} & \multicolumn{4}{|c|}{$262(12.86)$} \\
\hline \multicolumn{3}{|l|}{$500-1,999$} & \multicolumn{4}{|c|}{$619(30.39)$} \\
\hline \multicolumn{3}{|l|}{$\geq 2,000$} & \multicolumn{4}{|c|}{$1048(51.42)$} \\
\hline \multicolumn{7}{|c|}{ Dependent variables: Count Variables } \\
\hline \multirow{2}{*}{ Frequency } & \multicolumn{2}{|c|}{$\begin{array}{c}\text { Motorized Vehicle } \\
\text { Count }\end{array}$} & \multicolumn{2}{|c|}{$\begin{array}{c}\text { Number of } \\
\text { motorized tours }\end{array}$} & \multicolumn{2}{|c|}{$\begin{array}{l}\text { Number of non- } \\
\text { motorized tours }\end{array}$} \\
\hline & Number & $\%$ & Number & $\%$ & Number & $\%$ \\
\hline 0 & 84 & 4.12 & 78 & 3.83 & 1359 & 66.72 \\
\hline 1 & 516 & 25.33 & 539 & 26.46 & 415 & 20.37 \\
\hline 2 & 917 & 45.02 & 532 & 26.12 & 154 & 7.56 \\
\hline 3 & 357 & 17.53 & 346 & 16.99 & 59 & 2.90 \\
\hline 4 & 108 & 5.30 & 208 & 10.21 & 35 & 1.72 \\
\hline 5 & 38 & 1.87 & 116 & 5.69 & 9 & 0.44 \\
\hline 6 & 9 & 0.44 & 87 & 4.27 & 4 & 0.20 \\
\hline 7 & 4 & 0.20 & 48 & 2.36 & 1 & 0.05 \\
\hline 8 & 2 & 0.10 & 32 & 1.57 & 1 & 0.05 \\
\hline 9 & 1 & 0.05 & 22 & 1.08 & 0 & 0.00 \\
\hline 10 or more & 1 & 0.05 & 29 & 1.42 & 0 & 0.00 \\
\hline \multicolumn{7}{|c|}{ Dependent variables: Continuous Variable } \\
\hline Variable & Mean & & td. Dev. & Min. & & Max. \\
\hline $\begin{array}{l}\text { Natural logarithm of } \\
\text { average tour distance }\end{array}$ & 2.68 & & 1.36 & -2.30 & & 5.60 \\
\hline
\end{tabular}


Table 4. Model Estimation Result (variables significant at $5 \%$ level of significance unless otherwise mentioned)

\begin{tabular}{|c|c|c|c|c|c|c|c|c|}
\hline \multirow[b]{3}{*}{ Variables } & \multirow{2}{*}{\multicolumn{4}{|c|}{$\begin{array}{c}\text { MNP } \\
\text { Density Categories [households per sq. mile] }\end{array}$}} & \multicolumn{3}{|c|}{ Counts } & \multirow{3}{*}{$\begin{array}{c}\text { LR } \\
\begin{array}{c}\text { Natural Log. of } \\
\text { average tour } \\
\text { distance }\end{array}\end{array}$} \\
\hline & & & & & \multirow[b]{2}{*}{$\begin{array}{l}\text { Number of } \\
\text { vehicles }\end{array}$} & \multirow{2}{*}{$\begin{array}{l}\text { Number of } \\
\text { motorized } \\
\text { tours }\end{array}$} & \multirow{2}{*}{$\begin{array}{c}\text { Number of } \\
\text { non- } \\
\text { motorized } \\
\text { tours }\end{array}$} & \\
\hline & 0-99 & $100-499$ & $\begin{array}{c}500- \\
1,999\end{array}$ & $\geq 2,000$ & & & & \\
\hline Constant & -1.341 & -1.142 & -0.756 & - & 0.251 & 0.470 & -0.908 & 1.502 \\
\hline $\begin{array}{l}\text { Family structure variables } \\
\text { Single parent } \\
\text { Couple } \\
\text { Nuclear family } \\
\text { Joint family } \\
\end{array}$ & $\begin{array}{c}-0.961 \\
0.202 \\
- \\
- \\
\end{array}$ & $\begin{array}{c}- \\
0.202 \\
- \\
0.260 \\
\end{array}$ & $\begin{array}{l}- \\
- \\
- \\
-\end{array}$ & $\begin{array}{l}- \\
- \\
- \\
-\end{array}$ & $\begin{array}{l}0.167 \\
0.341 \\
0.363 \\
0.585 \\
\end{array}$ & $\begin{array}{l}0.587 \\
0.514 \\
1.065 \\
0.910 \\
\end{array}$ & $\begin{array}{l}0.703 \\
0.301 \\
1.116 \\
1.053 \\
\end{array}$ & $\begin{array}{l}0.232 \\
0.078^{\dagger \dagger} \\
\end{array}$ \\
\hline Natural Log. of income [US\$/year] & 0.177 & 0.168 & 0.256 & - & 0.266 & 0.123 & & 0.239 \\
\hline $\begin{array}{l}\text { Household race and ethnicity variables } \\
\text { Respondent race is Hispanic } \\
\text { Respondent race is Asian }\end{array}$ & $-\overline{-}$ & $-\overline{-}$ & $-\overline{193}$ & - & & -0.094 & -0.254 & $-0.152^{\dagger \dagger}$ \\
\hline $\begin{array}{l}\text { Highest education status variable } \\
\text { Highest education level is Bachelor's } \\
\text { degree or higher }\end{array}$ & $-0.142^{\dagger}$ & - & - & - & -0.104 & & 0.220 & \\
\hline $\begin{array}{l}\text { Immigrant variables } \\
\text { All immigrants household } \\
\text { Combination of immigrant and non- } \\
\text { immigrant household } \\
\end{array}$ & $\begin{array}{l}-0.350 \\
-0.238^{\dagger}\end{array}$ & - & - & - & & $0.059^{\dagger}$ & $0.237^{\dagger}$ & \\
\hline $\begin{array}{l}\text { Residential location (Density in housing } \\
\text { units per square mile) } \\
0-99 \\
100-499 \\
500-1,999 \\
\end{array}$ & & & & & $\begin{array}{l}0.146 \\
0.127 \\
0.088 \\
\end{array}$ & & & $\begin{array}{l}0.441 \\
0.419 \\
0.269 \\
\end{array}$ \\
\hline Number of vehicles & & & & & & 0.053 & -0.163 & 0.235 \\
\hline $\begin{array}{l}\text { Threshold parameters } \\
\varphi \text { (flexibility parameter) } \\
\theta \text { (dispersion parameter) } \\
\end{array}$ & & & & & $\begin{array}{c}0.576 \\
150.000^{*} \\
\end{array}$ & $\begin{array}{c}0.723 \\
150.000^{*} \\
\end{array}$ & $\begin{array}{l}0.000^{*} \\
0.908 \\
\end{array}$ & \\
\hline
\end{tabular}

"Not significant at $5 \%$ level of significance but significant at $15 \%$ level of significance

${ }^{\dagger}$ Not significant at $15 \%$ level of significance

${ }^{*}$ Not Estimated 
Table 5. Model Estimation Result - Covariance Matrix - Motorized Modes Priority (variables significant at $5 \%$ level of significance unless otherwise mentioned)

\begin{tabular}{|c|c|c|c|c|c|c|c|}
\hline \multirow{3}{*}{ Covariance Matrix } & \multirow{2}{*}{\multicolumn{3}{|c|}{$\begin{array}{c}\text { MNP } \\
\text { Density Categories } \\
\text { [households per sq. } \text { mile] }\end{array}$}} & \multicolumn{3}{|c|}{ Counts } & \multirow{3}{*}{$\begin{array}{c}\text { LR } \\
\begin{array}{c}\text { Natural Log. } \\
\text { of average } \\
\text { tour distance } \\
\text { [miles] }\end{array}\end{array}$} \\
\hline & & & & \multirow{2}{*}{$\begin{array}{l}\text { Number of } \\
\text { vehicles }\end{array}$} & \multirow{2}{*}{$\begin{array}{l}\text { Number of } \\
\text { motorized } \\
\text { tours }\end{array}$} & \multirow{2}{*}{$\begin{array}{l}\text { Number of } \\
\text { non- } \\
\text { motorized } \\
\text { tours }\end{array}$} & \\
\hline & $0-99$ & $100-499$ & $500-1,999$ & & & & \\
\hline 0-99 households per sq. mile & $1.000^{*}$ & & & & & & \\
\hline 100-499 households per sq. mile & $0.500^{*}$ & $1.000^{*}$ & & & & & \\
\hline 500-1,999 households per sq. mile & $0.500^{*}$ & $0.500^{*}$ & $1.000^{*}$ & & & & \\
\hline Number of vehicles & $0.000^{*}$ & 0.052 & 0.073 & $1.000^{*}$ & & & \\
\hline Number of motorized tours & $0.000^{*}$ & $0.000^{*}$ & $0.000^{*}$ & 0.176 & $1.000^{*}$ & & \\
\hline Number of non-motorized tours & $0.000^{*}$ & $0.000^{*}$ & $0.000^{*}$ & -0.079 & -0.237 & $1.000^{*}$ & \\
\hline Natural Log. of average tour distance & $0.000^{*}$ & $0.000^{*}$ & $0.000^{*}$ & $0.000^{*}$ & $0.000^{*}$ & $0.000^{*}$ & 1.636 \\
\hline
\end{tabular}

${ }^{\dagger}$ Not significant at $5 \%$ level of significance but significant at $15 \%$ level of significance

${ }^{\dagger}$ Not significant at $15 \%$ level of significance

${ }^{*}$ Not Estimated 
Table 6. Treatment Effects Corresponding to Transplanting a Random Household from a 500-1999 HHs per Sq. Mile Density Neighborhood to the Highest Density Neighborhood

\begin{tabular}{|c|c|c|c|c|}
\hline \multirow{2}{*}{ Variable } & \multirow{2}{*}{$\begin{array}{c}\text { ATE from Joint } \\
\text { Model }\end{array}$} & \multirow{2}{*}{$\begin{array}{c}\text { ATE from } \\
\text { Independent } \\
\text { Model }\end{array}$} & \multicolumn{2}{|c|}{$\%$ Difference Attributable to } \\
\hline & & & "True" Effect & $\begin{array}{l}\text { Self-Selection } \\
\text { Effect }\end{array}$ \\
\hline Motorized vehicle ownership & -0.189 & -0.261 & 72 & 28 \\
\hline Number of motorized tours & -0.038 & -0.068 & 56 & 44 \\
\hline Number of non-motorized tours & 0.016 & 0.027 & 59 & 41 \\
\hline Average tour distance & -2.250 & -3.083 & 73 & 27 \\
\hline Vehicle miles of travel & -11.437 & -15.960 & 72 & 28 \\
\hline
\end{tabular}

